\title{
Combination-Combination Synchronization of Four Nonlinear Complex Chaotic Systems
}

\author{
Xiaobing Zhou, ${ }^{1}$ Lianglin Xiong, ${ }^{2}$ and Xiaomei Cai ${ }^{3}$ \\ ${ }^{1}$ School of Information Science and Engineering, Yunnan University, Kunming 650091, China \\ ${ }^{2}$ School of Mathematics and Computer Science, Yunnan University of Nationalities, Kunming 650031, China \\ ${ }^{3}$ Bureau of Asset Management, Yunnan University, Kunming 650091, China \\ Correspondence should be addressed to Xiaobing Zhou; zhouxb.cn@gmail.com
}

Received 13 August 2013; Revised 25 October 2013; Accepted 30 October 2013; Published 3 February 2014

Academic Editor: Narcisa C. Apreutesei

Copyright (C) 2014 Xiaobing Zhou et al. This is an open access article distributed under the Creative Commons Attribution License, which permits unrestricted use, distribution, and reproduction in any medium, provided the original work is properly cited.

\begin{abstract}
This paper investigates the combination-combination synchronization of four nonlinear complex chaotic systems. Based on the Lyapunov stability theory, corresponding controllers to achieve combination-combination synchronization among four different nonlinear complex chaotic systems are derived. The special cases, such as combination synchronization and projective synchronization, are studied as well. Numerical simulations are given to illustrate the theoretical analysis.
\end{abstract}

\section{Introduction}

In 1982, Fowler et al. [1] generalized the real Lorenz model to a complex Lorenz model, which can be used to describe and simulate the physics of a detuned laser and the thermal convection of liquid flows [2,3]. After that, many new chaotic and hyperchaotic complex systems have been reported and intensively studied, including the complex Van der Pol oscillators [4], the complex Chen and complex Lü systems [5], complex detuned laser system [6], complex hyperchaotic Lorenz system [7], complex modified hyperchaotic Lü system [8], and a novel hyperchaotic complex-variable system [9] which generates 2-, 3-, and 4-scroll attractors.

Since Pecora and Carroll [10] first proposed the driveresponse concept for constructing synchronization of coupled chaotic systems, synchronization in chaotic systems has been extensively investigated due to their potential applications in the fields of secure communications; optical, chemical, physical, and biological systems; neural networks; and so forth [11-13]. When applying the complex systems in communications, the complex variables will double the number of variables and can increase the content and security of the transmitted information. Based on the Lyapunov stability theory, linear feedback controller was derived to achieve hybrid projective synchronization in a chaotic complex nonlinear system [14]. The authors [15] achieved adaptive antisynchronization of a class of chaotic complex nonlinear systems described by a united mathematical expression with fully uncertain parameters. In [16], the author investigated the modified projective phase synchronization of chaotic complex nonlinear systems. Based on the passive theory, the authors studied the projective synchronization of hyperchaotic complex nonlinear systems and its application in secure communications [17]. In [18], the authors achieved fast synchronization of a novel hyperchaotic complex system based on finite-time stability theory.

However, most of the existing synchronization schemes are based on the usual drive-response synchronization mode, which has one drive system and one response system. In [19], Luo et al. proposed the combination synchronization scheme, which has two drive systems and one response system. Zhou et al. investigated combination synchronization of three nonlinear complex hyperchaotic systems in [20]. Sun et al. [21] extended the combination synchronization scheme to the combination-combination synchronization scheme, where synchronization is achieved between two drive systems and two response systems. This synchronization scheme has advantages over the other synchronization schemes, such that it can provide greater security in secure communication. 
For the nonlinear complex chaotic or hyperchaotic systems, there are no work on combination-combination synchronization for them. This paper aims to study the combination-combination synchronization of four nonlinear complex chaotic systems. The rest of this paper is organized as follows. Section 2 introduces the scheme of combinationcombination synchronization. In Section 3, we investigate combination-combination synchronization of four complex nonlinear chaotic systems. Numerical simulations are conducted in Section 4. Finally, conclusions are given in Section 5 .

\section{The Scheme of Combination-Combination Synchronization}

In the scheme of combination-combination synchronization, there are four nonlinear dynamical systems, two drive systems, and two response system.

The two drive systems are, respectively, given by

$$
\begin{aligned}
& \dot{x}_{1}=f_{1}\left(x_{1}\right), \\
& \dot{x}_{2}=f_{2}\left(x_{2}\right) .
\end{aligned}
$$

The two response systems are, respectively, described by

$$
\begin{aligned}
& \dot{y}_{1}=g_{1}\left(y_{1}\right)+\varphi, \\
& \dot{y}_{2}=g_{2}\left(y_{2}\right)+\varphi^{*},
\end{aligned}
$$

where $x_{1}=\left(x_{11}, x_{12}, \ldots, x_{1 n}\right)^{T}, x_{2}=\left(x_{21}, x_{22}, \ldots, x_{2 n}\right)^{T}$, $y_{1}=\left(y_{11}, y_{12}, \ldots, y_{1 n}\right)^{T}$, and $y_{2}=\left(y_{21}, y_{22}, \ldots, y_{2 n}\right)^{T}$ are the state vectors of the systems (1), (2), (3), and (4), respectively; $f_{1}(\cdot), f_{2}(\cdot), g_{1}(\cdot), g_{2}(\cdot): R^{n} \rightarrow R^{n}$ are four continuous vector functions and $\varphi, \varphi^{*}: R^{n} \times R^{n} \times R^{n} \times R^{n} \rightarrow R^{n}$ are two controller vectors which will be designed.

Definition 1 (see [21]). If there exist four constant matrices $A$, $B, C$, and $D \in R^{n}$ and $C \neq 0$ or $D \neq 0$ such that

$$
\lim _{t \rightarrow+\infty}\left\|A x_{1}+B x_{2}-C y_{1}-D y_{2}\right\|=0
$$

the drive systems (1) and (2) are realized combination-combination synchronization with the response systems (3) and (4), where $\|\cdot\|$ represents the matrix norm.

Remark 2. The combination-combination synchronization can be reduced to combination synchronization, projective synchronization, and even control problem, if we choose specific values of $A, B, C$, and $D$.

\section{Combination-Combination Synchronization of Four Nonlinear Complex Chaotic Systems}

In this section, we investigate the combination-combination synchronization of four nonlinear complex chaotic systems.
The first drive system [22] is given by

$$
\begin{gathered}
\dot{x}_{11}=\alpha_{1}\left(x_{12}-x_{11}\right)+x_{12} x_{13}, \\
\dot{x}_{12}=\gamma_{1} x_{11}-x_{12}-x_{11} x_{13}, \\
\dot{x}_{13}=-\beta_{1} x_{13}+\frac{1}{2}\left(\bar{x}_{11} x_{12}+x_{11} \bar{x}_{12}\right),
\end{gathered}
$$

and the second drive system [23] is described as follows:

$$
\begin{gathered}
\dot{x}_{21}=a_{1} x_{21}+b_{1} x_{22} x_{23}, \\
\dot{x}_{22}=a_{2} x_{22}+b_{2} x_{21} x_{23}, \\
\dot{x}_{23}=a_{3} x_{23}+\frac{b_{3}}{2}\left(\bar{x}_{21} x_{22}+x_{21} \bar{x}_{22}\right) .
\end{gathered}
$$

The first response system [6] takes the following form:

$$
\begin{gathered}
\dot{y}_{11}=\sigma_{3} y_{12}-\sigma_{3}\left(1-i \delta_{3}\right) y_{11}+\varphi_{1}+i \varphi_{2}, \\
\dot{y}_{12}=\left(\alpha_{3}-y_{13}\right) y_{11}-\left(1+i \delta_{3}\right) y_{12}+\varphi_{3}+i \varphi_{4}, \\
\dot{y}_{13}=-\beta_{3} y_{13}+\frac{1}{2}\left(\bar{y}_{11} y_{12}+y_{11} \bar{y}_{12}\right)+\varphi_{5},
\end{gathered}
$$

and the second response [9] is given by

$$
\begin{gathered}
\dot{y}_{21}=y_{22}-\alpha_{4} y_{21}+\beta_{4} y_{22} y_{23}+\varphi_{1}^{*}+i \varphi_{2}^{*}, \\
\dot{y}_{22}=\gamma_{4} y_{22}-y_{21} y_{23}+y_{23}+\varphi_{3}^{*}+i \varphi_{4}^{*}, \\
\dot{y}_{23}=\frac{\delta_{4}}{2}\left(\bar{y}_{21} y_{22}+y_{21} \bar{y}_{22}\right)-\sigma_{4} y_{23}+\varphi_{5}^{*},
\end{gathered}
$$

where $\alpha_{1}, \beta_{1}, \gamma_{1}, a_{1}, a_{2}, a_{3}, b_{1}, b_{2}, b_{3}, \alpha_{3}, \beta_{3}, \delta_{3}, \sigma_{3}, \alpha_{4}, \beta_{4}, \gamma_{4}, \sigma_{4}$, and $\delta_{4}$ are system parameters; $x_{11}=u_{1}+i u_{2}, x_{12}=u_{3}+i u_{4}$, $x_{21}=v_{1}+i v_{2}, x_{22}=v_{3}+i v_{4}, y_{11}=w_{1}+i w_{2}, y_{12}=w_{3}+i w_{4}$, $y_{21}=\mu_{1}+i \mu_{2}$, and $y_{22}=\mu_{3}+i \mu_{4}$ are complex variables; $i=$ $\sqrt{-1}$; and $u_{i}, v_{i}, w_{i}, \mu_{i}(i=1,2,3,4), x_{13}=u_{5}, x_{23}=v_{5}, y_{13}=$ $w_{5}$, and $y_{23}=\mu_{5}$ are real variables. The overbar represents complex conjugate function. $\varphi_{i}$ and $\varphi_{i}^{*}(i=1,2,3,4,5)$ are real controllers to be determined. Their chaotic attractors are illustrated in Figures 1, 2, 3, and 4, respectively.

For the convenience of our discussions, we assume $A=$ $\operatorname{diag}\left(k_{1}, k_{2}, k_{3}\right), B=\operatorname{diag}\left(l_{1}, l_{2}, l_{3}\right), C=\operatorname{diag}\left(m_{1}, m_{2}, m_{3}\right)$, and $D=\operatorname{diag}\left(n_{1}, n_{2}, n_{3}\right)$ in our synchronization scheme.

We define error states between the drive systems (6) and (7) and the response systems (8) and (9) as

$$
\begin{gathered}
e_{1}+i e_{2}=k_{1} x_{11}+l_{1} x_{21}-m_{1} y_{11}-n_{1} y_{21}, \\
e_{3}+i e_{4}=k_{2} x_{12}+l_{2} x_{22}-m_{2} y_{12}-n_{2} y_{22}, \\
e_{5}=k_{3} x_{13}+l_{3} x_{23}-m_{3} y_{13}-n_{3} y_{23},
\end{gathered}
$$

such that

$$
\begin{aligned}
& \lim _{t \rightarrow \infty}\left\|k_{1} x_{11}+l_{1} x_{21}-m_{1} y_{11}-n_{1} y_{21}\right\|=0, \\
& \lim _{t \rightarrow \infty}\left\|k_{2} x_{12}+l_{2} x_{22}-m_{2} y_{12}-n_{2} y_{22}\right\|=0, \\
& \lim _{t \rightarrow \infty}\left\|k_{3} x_{13}+l_{3} x_{23}-m_{3} y_{13}-n_{3} y_{23}\right\|=0 .
\end{aligned}
$$




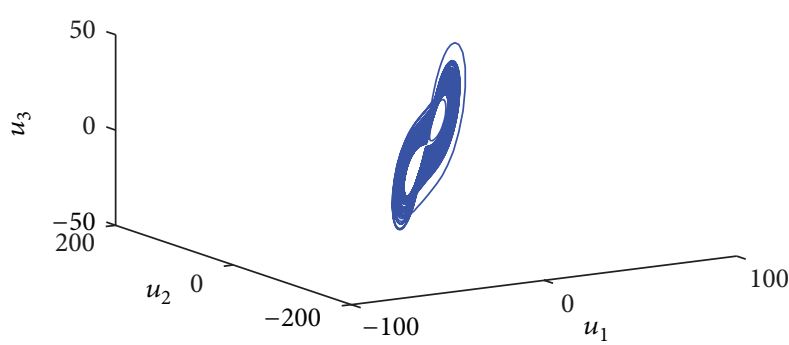

(a)

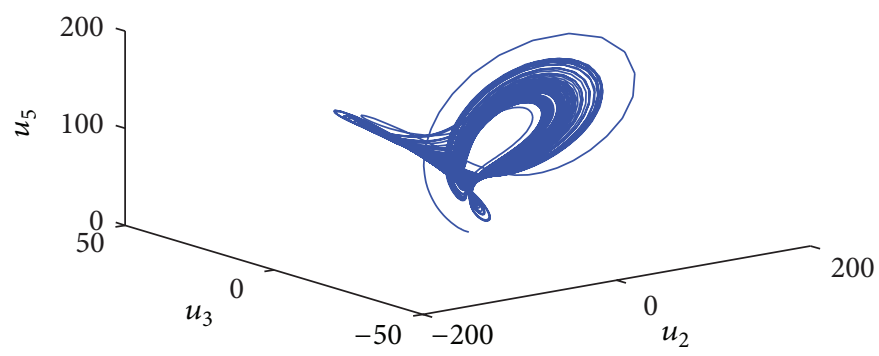

(c)

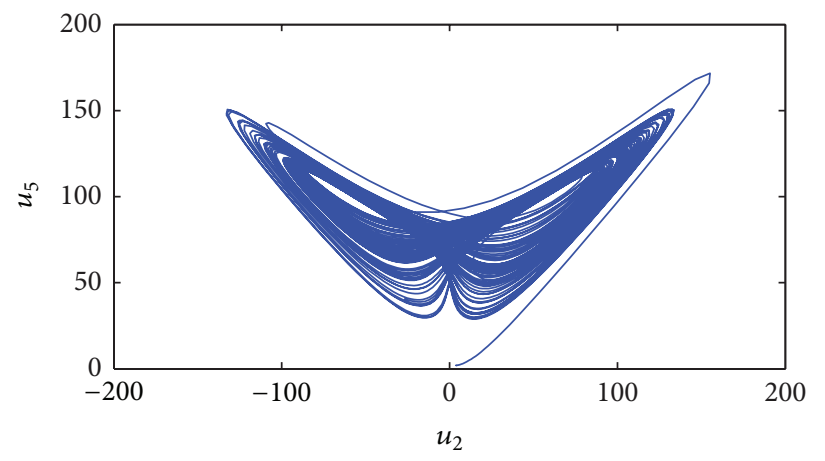

(e)

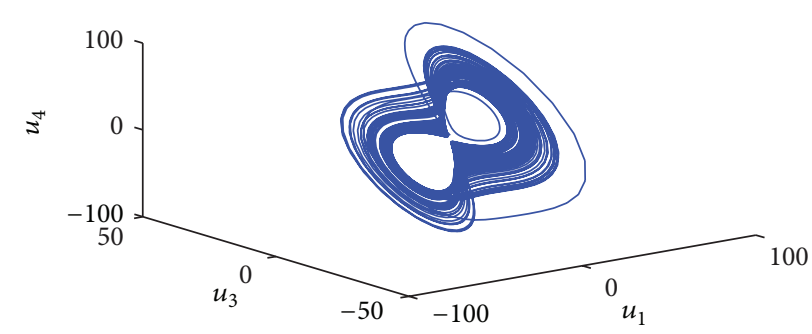

(b)

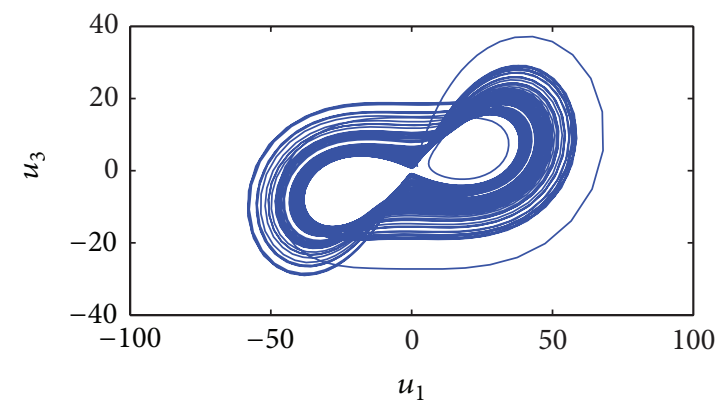

(d)

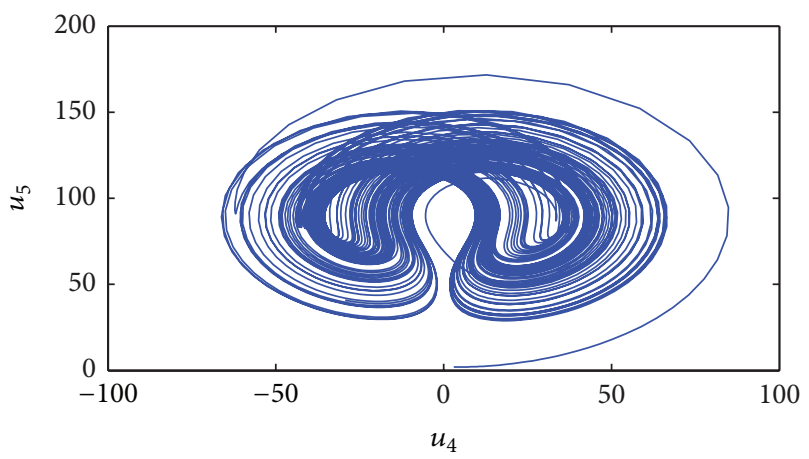

(f)

FIGURE 1: Chaotic attractor for system (6). (a)-(c) Projections in 3D space; (d)-(f) projections in 2D plane.

Thus, we have the following error dynamical system:

$$
\begin{gathered}
\dot{e}_{1}+i \dot{e}_{2}=k_{1} \dot{x}_{11}+l_{1} \dot{x}_{21}-m_{1} \dot{y}_{11}-n_{1} \dot{y}_{21}, \\
\dot{e}_{3}+i \dot{e}_{4}=k_{2} \dot{x}_{12}+l_{2} \dot{x}_{22}-m_{2} \dot{y}_{12}-n_{2} \dot{y}_{22}, \\
\dot{e}_{5}=k_{3} \dot{x}_{13}+l_{3} \dot{x}_{23}-m_{3} \dot{y}_{13}-n_{3} \dot{y}_{23} .
\end{gathered}
$$

Substituting (6)-(9) into (12) and separating the real and imaginary parts yields

$$
\begin{aligned}
\dot{e}_{1}= & k_{1}\left[\alpha_{1}\left(u_{3}-u_{1}\right)+u_{3} u_{5}\right]+l_{1}\left(a_{1} v_{1}+b_{1} v_{3} v_{5}\right) \\
& -m_{1}\left[\sigma_{3}\left(w_{3}-w_{1}-\delta_{3} w_{2}\right)+\varphi_{1}\right] \\
& -n_{1}\left(\mu_{3}-\alpha_{4} \mu_{1}+\beta_{4} \mu_{3} \mu_{5}+\varphi_{1}^{*}\right), \\
\dot{e}_{2}= & k_{1}\left[\alpha_{1}\left(u_{4}-u_{2}\right)+u_{4} u_{5}\right]+l_{1}\left(a_{1} v_{2}+b_{1} v_{4} v_{5}\right) \\
& -m_{1}\left[\sigma_{3}\left(w_{4}-w_{2}+\delta_{3} w_{1}\right)+\varphi_{2}\right] \\
& -n_{1}\left(\mu_{4}-\alpha_{4} \mu_{2}+\beta_{4} \mu_{4} \mu_{5}+\varphi_{2}^{*}\right),
\end{aligned}
$$

$$
\begin{aligned}
\dot{\mathbf{e}}_{3}=\mathbf{k}_{\mathbf{2}}\left(\boldsymbol{\gamma}_{\mathbf{1}} \mathbf{u}_{\mathbf{1}}-\mathbf{u}_{\mathbf{5}} \mathbf{u}_{\mathbf{1}}-\mathbf{u}_{\mathbf{3}}\right)+\mathbf{l}_{\mathbf{2}}\left(\mathbf{a}_{\mathbf{2}} \mathbf{v}_{\mathbf{3}}+\mathbf{b}_{\mathbf{2}} \mathbf{v}_{\mathbf{1}} \mathbf{v}_{\mathbf{5}}\right) \\
-\mathbf{m}_{\mathbf{2}}\left[\left(\boldsymbol{\alpha}_{\mathbf{3}}-\mathbf{w}_{\mathbf{5}}\right) \mathbf{w}_{\mathbf{1}}-\mathbf{w}_{\mathbf{3}}-\boldsymbol{\delta}_{\mathbf{3}} \mathbf{w}_{\mathbf{4}}+\boldsymbol{\varphi}_{3}\right] \\
-\mathbf{n}_{\mathbf{2}}\left(\boldsymbol{\gamma}_{4} \boldsymbol{\mu}_{\mathbf{3}}-\boldsymbol{\mu}_{\mathbf{1}} \boldsymbol{\mu}_{\mathbf{5}}+\boldsymbol{\mu}_{\mathbf{5}}+\boldsymbol{\varphi}_{3}^{*}\right), \\
\dot{e}_{4}=k_{2}\left(\gamma_{1} u_{2}-u_{5} u_{2}-u_{4}\right)+l_{2}\left(a_{2} v_{4}+b_{2} v_{2} v_{5}\right) \\
-m_{2}\left[\left(\alpha_{3}-w_{5}\right) w_{2}-w_{4}-\delta_{3} w_{3}+\varphi_{4}\right] \\
-n_{2}\left(\gamma_{4} \mu_{4}-\mu_{2} \mu_{5}+\varphi_{4}^{*}\right), \\
\dot{e}_{5}=k_{3}\left(-\beta_{1} u_{5}+u_{1} u_{3}+u_{2} u_{4}\right) \\
+l_{3}\left[a_{3} v_{5}+b_{3}\left(v_{1} v_{3}+v_{2} v_{4}\right)\right] \\
-m_{3}\left(-\beta_{3} w_{5}+w_{1} w_{3}+w_{2} w_{4}+\varphi_{5}\right) \\
-n_{3}\left[\delta_{4}\left(\mu_{1} \mu_{3}+\mu_{2} \mu_{4}\right)-\sigma_{4} \mu_{5}+\varphi_{5}^{*}\right] .
\end{aligned}
$$

Denote $U_{i}=m_{i} \varphi_{i}+n_{i} \varphi_{i}^{*}(i=1,2,3,4,5)$; then we obtain the following results. 


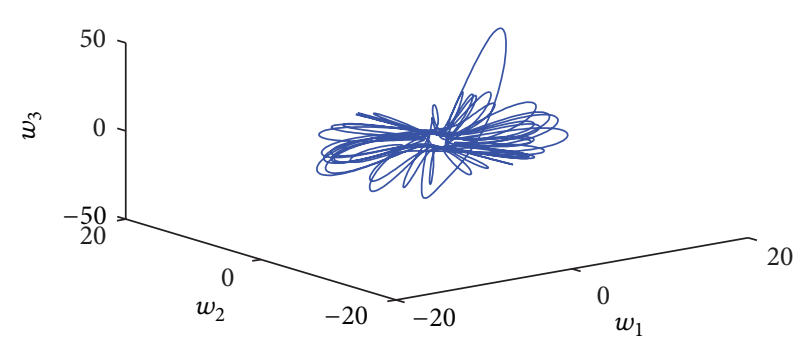

(a)

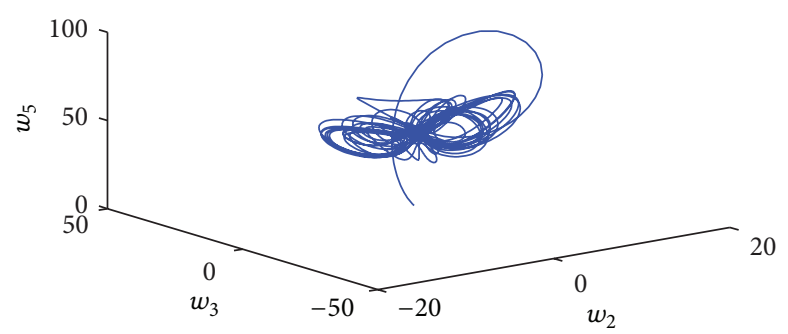

(c)

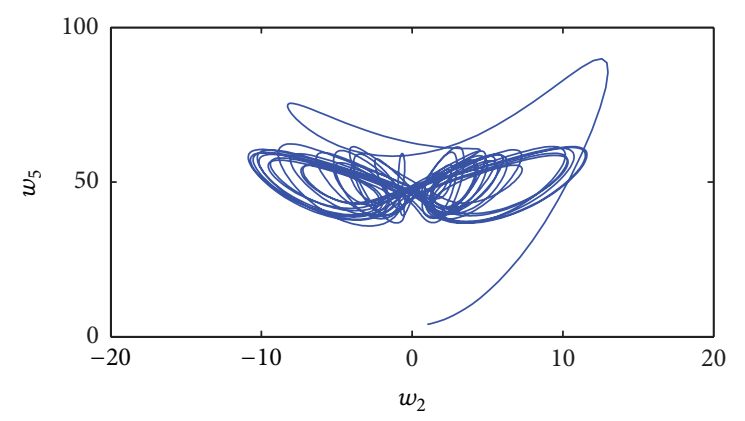

(e)

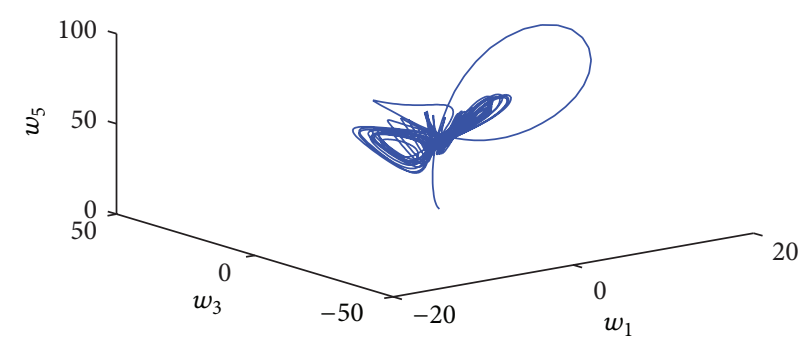

(b)

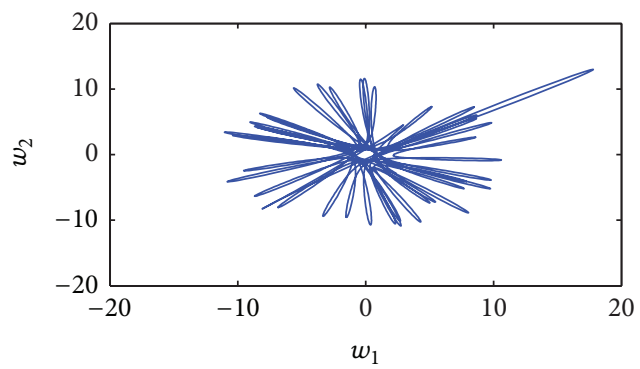

(d)

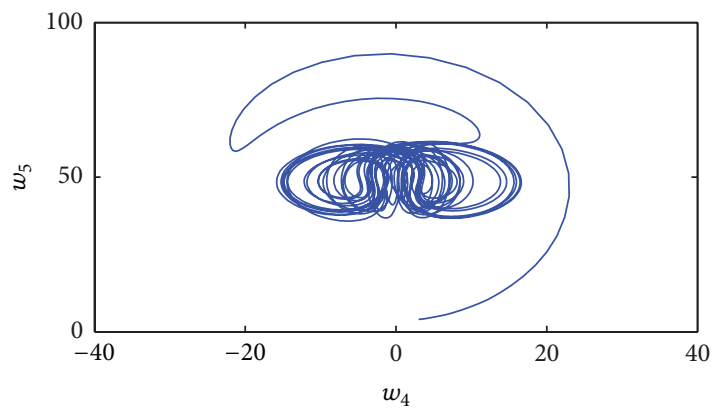

(f)

Figure 2: Chaotic attractor for system (7). (a)-(c) Projections in 3D space; (d)-(f) projections in 2D plane.

Theorem 3. If the controllers are chosen as follows:

$$
\begin{aligned}
U_{1}= & k_{1} u_{1}+l_{1} v_{1}-m_{1} w_{1}-n_{1} \mu_{1} \\
& +a_{1}\left(k_{1} u_{2}+l_{1} v_{2}-m_{1} w_{2}-n_{1} \mu_{2}\right) \\
& +k_{1}\left[\alpha_{1}\left(u_{3}-u_{1}\right)+u_{3} u_{5}\right]+l_{1}\left(a_{1} v_{1}+b_{1} v_{3} v_{5}\right) \\
& -m_{1} \sigma_{3}\left(w_{3}-w_{1}-\delta_{3} w_{2}\right)-n_{1}\left(\mu_{3}-\alpha_{4} \mu_{1}+\beta_{4} \mu_{3} \mu_{5}\right),
\end{aligned}
$$$$
\mathrm{U}_{2}=\mathbf{k}_{1} \mathbf{u}_{2}+\mathbf{l}_{1} \mathbf{v}_{2}-\mathbf{m}_{1} \mathbf{w}_{2}-\mathbf{n}_{1} \mu_{2}
$$$$
-a_{1}\left(k_{1} u_{1}+l_{1} v_{1}-m_{1} w_{1}-n_{1} \mu_{1}\right)
$$$$
-a_{2}\left(k_{2} u_{3}+l_{2} v_{3}-m_{2} w_{3}-n_{2} \mu_{3}\right)
$$$$
+\mathbf{k}_{1}\left[\alpha_{1}\left(\mathbf{u}_{4}-\mathbf{u}_{2}\right)+\mathbf{u}_{4} \mathbf{u}_{5}\right]+\mathbf{l}_{1}\left(\mathbf{a}_{1} \mathbf{v}_{2}+b_{1} \mathbf{v}_{4} \mathbf{v}_{5}\right)
$$$$
-\mathbf{m}_{1} \sigma_{3}\left(\mathbf{w}_{4}-\mathbf{w}_{2}+\delta_{3} \mathbf{w}_{1}\right)-\mathbf{n}_{1}\left(\mu_{4}-\alpha_{4} \mu_{2}+\beta_{4} \mu_{4} \mu_{5}\right),
$$$$
U_{3}=k_{2} u_{3}+l_{2} v_{3}-m_{2} w_{3}-n_{2} \mu_{3}
$$$$
+a_{2}\left(k_{1} u_{2}+l_{1} v_{2}-m_{1} w_{2}-n_{1} \mu_{2}\right)
$$$$
+a_{3}\left(k_{2} u_{4}+l_{2} v_{4}-m_{2} w_{4}-n_{2} \mu_{4}\right)
$$

$$
\begin{aligned}
& +k_{2}\left(\gamma_{1} u_{1}-u_{5} u_{1}-u_{3}\right)+l_{2}\left(a_{2} v_{3}+b_{2} v_{1} v_{5}\right) \\
& -m_{2}\left[\left(\alpha_{3}-w_{5}\right) w_{1}-w_{3}-\delta_{3} w_{4}\right] \\
& -n_{2}\left(\gamma_{4} \mu_{3}-\mu_{1} \mu_{5}+\mu_{5}\right) \text {, } \\
& \mathrm{U}_{4}=\mathbf{k}_{2} \mathbf{u}_{4}+\mathbf{l}_{2} \mathbf{v}_{4}-\mathbf{m}_{2} \mathbf{w}_{4}-\mathbf{n}_{2} \mu_{4} \\
& -a_{3}\left(k_{2} u_{3}+l_{2} v_{3}-m_{2} w_{3}-n_{2} \mu_{3}\right) \\
& -b_{1}\left(k_{3} u_{5}+l_{3} v_{5}-m_{3} w_{5}-n_{3} \mu_{5}\right) \\
& +\mathbf{k}_{2}\left(\gamma_{1} \mathbf{u}_{2}-\mathbf{u}_{5} \mathbf{u}_{2}-\mathbf{u}_{4}\right)+\mathbf{l}_{2}\left(\mathbf{a}_{2} \mathbf{v}_{4}+\mathbf{b}_{2} \mathbf{v}_{2} \mathbf{v}_{5}\right) \\
& -\mathbf{m}_{2}\left[\left(\alpha_{3}-\mathbf{w}_{5}\right) \mathbf{w}_{2}-\mathbf{w}_{4}-\delta_{3} \mathbf{w}_{3}\right] \\
& -\mathbf{n}_{2}\left(\gamma_{4} \mu_{4}-\mu_{2} \mu_{5}\right) \text {, } \\
& U_{5}=k_{3} u_{5}+l_{3} v_{5}-m_{3} w_{5}-n_{3} \mu_{5} \\
& +b_{1}\left(k_{2} u_{4}+l_{2} v_{4}-m_{2} w_{4}-n_{2} \mu_{4}\right) \\
& +k_{3}\left(-\beta_{1} u_{5}+u_{1} u_{3}+u_{2} u_{4}\right) \\
& +l_{3}\left[a_{3} v_{5}+b_{3}\left(v_{1} v_{3}+v_{2} v_{4}\right)\right]
\end{aligned}
$$




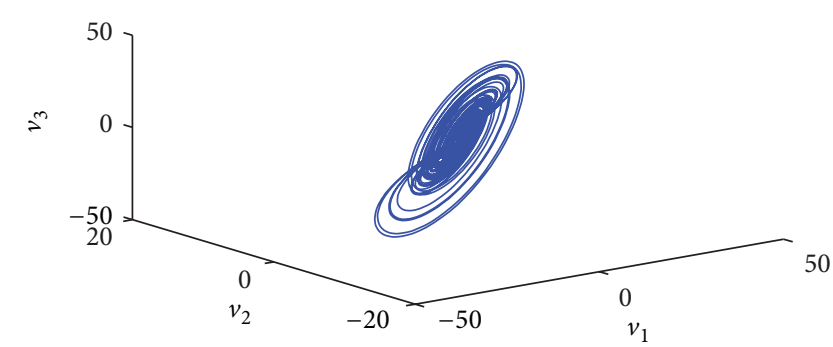

(a)

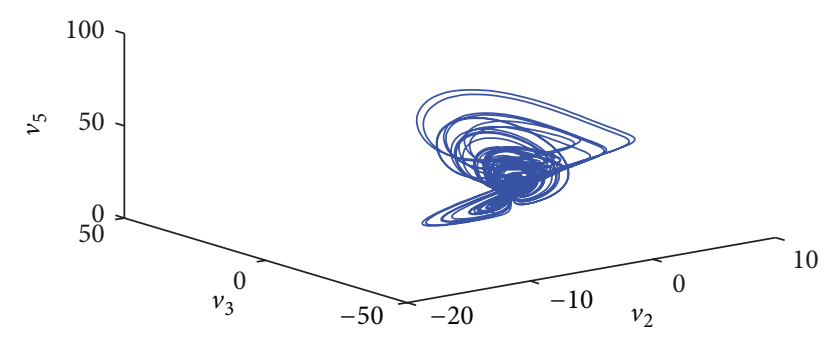

(c)

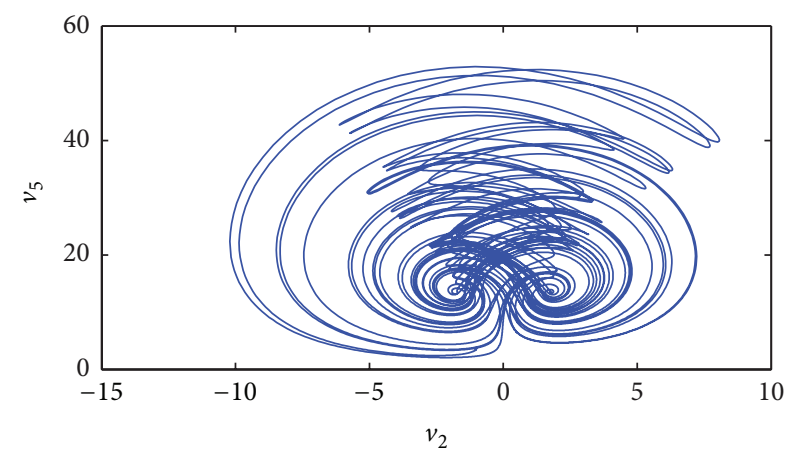

(e)

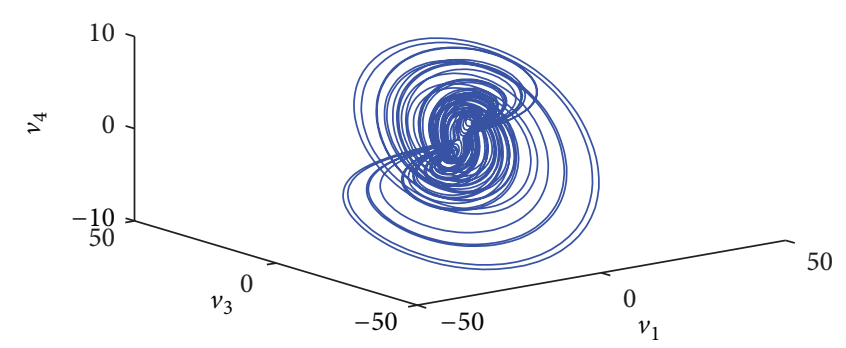

(b)

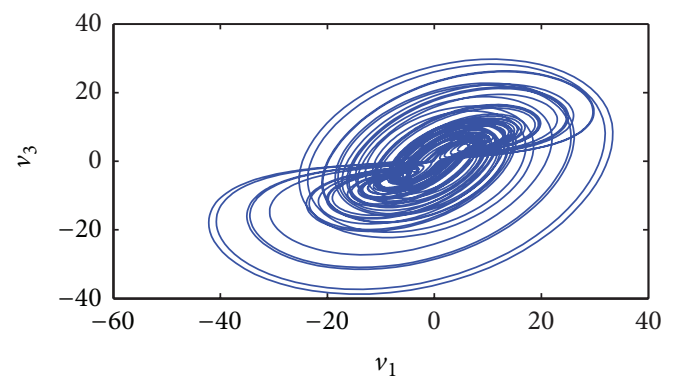

(d)

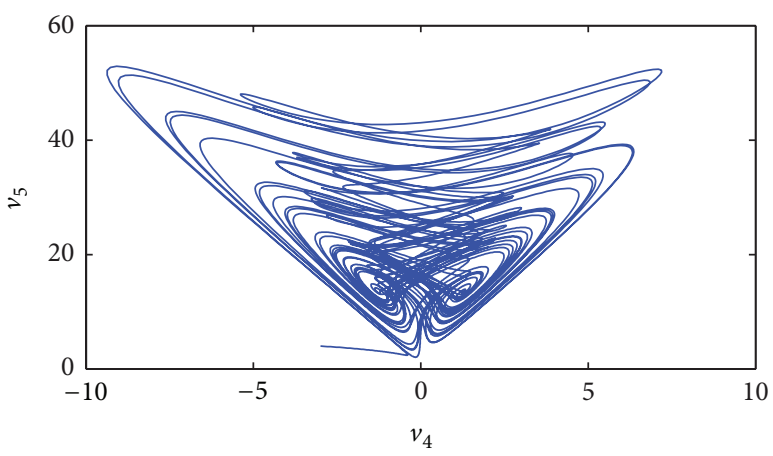

(f)

FIgure 3: Chaotic attractor for system (8). (a)-(c) Projections in 3D space; (d)-(f) projections in 2D plane.

$$
\begin{aligned}
& -m_{3}\left(-\beta_{3} w_{5}+w_{1} w_{3}+w_{2} w_{4}\right) \\
& -n_{3}\left[\delta_{4}\left(\mu_{1} \mu_{3}+\mu_{2} \mu_{4}\right)-\sigma_{4} \mu_{5}\right],
\end{aligned}
$$

then the drive systems (6) and (7) will achieve combinationcombination synchronization with the response systems (8) and (9).

Proof. Construct the following Lyapunov function:

$$
V=\frac{1}{2}\left(e_{1}^{2}+e_{2}^{2}+e_{3}^{2}+e_{4}^{2}+e_{5}^{2}\right)
$$

Taking the time derivative of $V$ along the trajectory of the error dynamical system (13) yields

$$
\begin{aligned}
& \dot{V}=e_{1} \dot{e}_{1}+e_{2} \dot{e}_{2}+e_{3} \dot{e}_{3}+e_{4} \dot{e}_{4}+e_{5} \dot{e}_{5} \\
&=e_{1}\left\{k_{1}\left[\alpha_{1}\left(u_{3}-u_{1}\right)+u_{3} u_{5}\right]+l_{1}\left(a_{1} v_{1}+b_{1} v_{3} v_{5}\right)\right. \\
&-m_{1}\left[\sigma_{3}\left(w_{3}-w_{1}-\delta_{3} w_{2}\right)+\varphi_{1}\right] \\
&\left.-n_{1}\left(\mu_{3}-\alpha_{4} \mu_{1}+\beta_{4} \mu_{3} \mu_{5}+\varphi_{1}^{*}\right)\right\}
\end{aligned}
$$

$$
\begin{aligned}
&+e_{2}\left\{k_{1}\left[\alpha_{1}\left(u_{4}-u_{2}\right)+u_{4} u_{5}\right]\right. \\
&+ l_{1}\left(a_{1} v_{2}+b_{1} v_{4} v_{5}\right) \\
&-m_{1}\left[\sigma_{3}\left(w_{4}-w_{2}+\delta_{3} w_{1}\right)+\varphi_{2}\right] \\
&\left.-n_{1}\left(\mu_{4}-\alpha_{4} \mu_{2}+\beta_{4} \mu_{4} \mu_{5}+\varphi_{2}^{*}\right)\right\} \\
&+e_{3}\left\{k_{2}\left(\gamma_{1} u_{1}-u_{5} u_{1}-u_{3}\right)+l_{2}\left(a_{2} v_{3}+b_{2} v_{1} v_{5}\right)\right. \\
&-m_{2}\left[\left(\alpha_{3}-w_{5}\right) w_{1}-w_{3}-\delta_{3} w_{4}+\varphi_{3}\right] \\
&\left.-n_{2}\left(\gamma_{4} \mu_{3}-\mu_{1} \mu_{5}+\mu_{5}+\varphi_{3}^{*}\right)\right\} \\
&+e_{4}\left\{k_{2}\left(\gamma_{1} u_{2}-u_{5} u_{2}-u_{4}\right)+l_{2}\left(a_{2} v_{4}+b_{2} v_{2} v_{5}\right)\right. \\
&-m_{2}\left[\left(\alpha_{3}-w_{5}\right) w_{2}-w_{4}-\delta_{3} w_{3}+\varphi_{4}\right] \\
&\left.-n_{2}\left(\gamma_{4} \mu_{4}-\mu_{2} \mu_{5}+\varphi_{4}^{*}\right)\right\} \\
&+e_{5}\left\{k_{3}\left(-\beta_{1} u_{5}+u_{1} u_{3}+u_{2} u_{4}\right)\right. \\
&+l_{3}\left[a_{3} v_{5}+b_{3}\left(v_{1} v_{3}+v_{2} v_{4}\right)\right]
\end{aligned}
$$




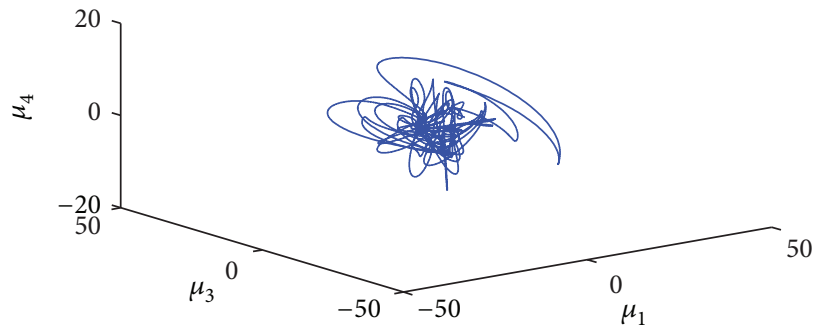

(a)

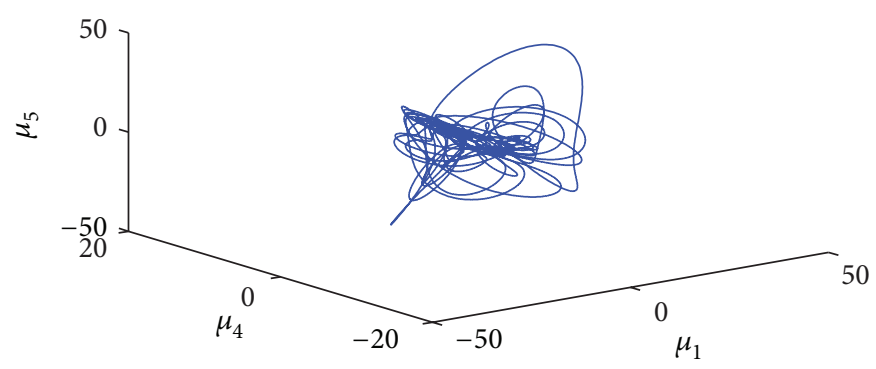

(c)

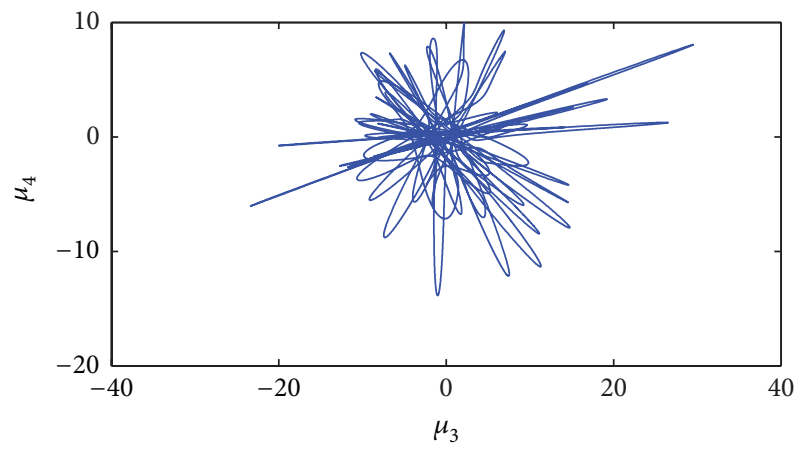

(e)

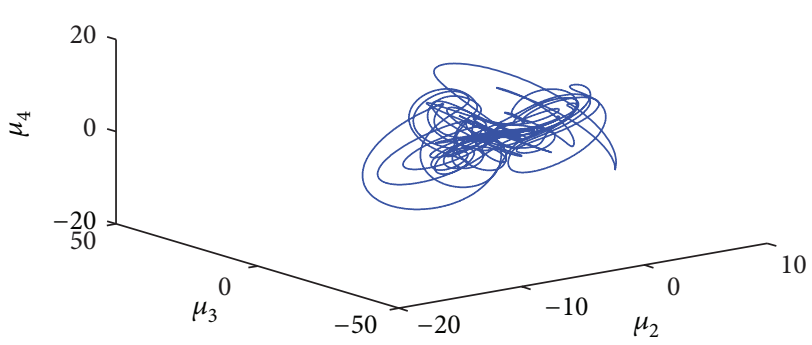

(b)

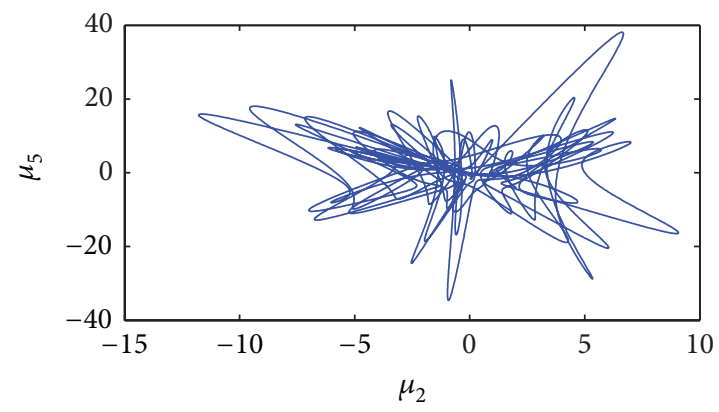

(d)

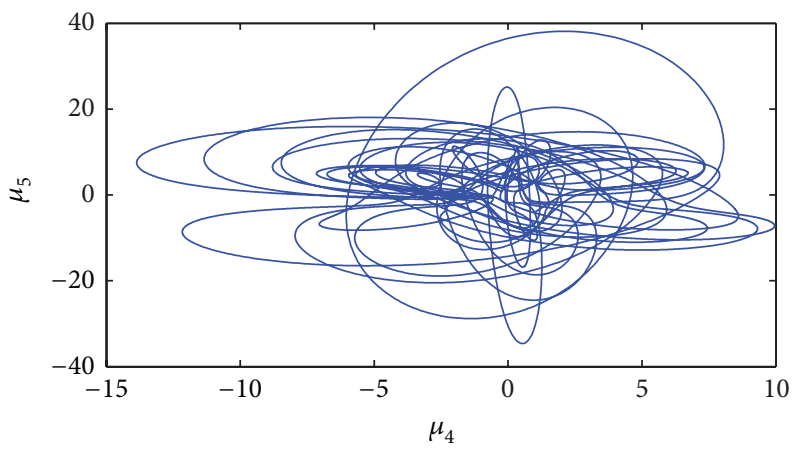

(f)

FIGURE 4: Chaotic attractor for system (9). (a)-(c) Projections in 3D space; (d)-(f) projections in 2D plane.

$$
\begin{gathered}
-m_{3}\left(-\beta_{3} w_{5}+w_{1} w_{3}+w_{2} w_{4}+\varphi_{5}\right) \\
\left.-n_{3}\left[\delta_{4}\left(\mu_{1} \mu_{3}+\mu_{2} \mu_{4}\right)-\sigma_{4} \mu_{5}+\varphi_{5}^{*}\right]\right\} \\
=e_{1}\left\{k_{1}\left[\alpha_{1}\left(u_{3}-u_{1}\right)+u_{3} u_{5}\right]+l_{1}\left(a_{1} v_{1}+b_{1} v_{3} v_{5}\right)\right. \\
-m_{1}\left[\sigma_{3}\left(w_{3}-w_{1}-\delta_{3} w_{2}\right)\right] \\
-n_{1}\left(\mu_{3}-\alpha_{4} \mu_{1}+\beta_{4} \mu_{3} \mu_{5}\right) \\
\left.-\left(m_{1} \varphi_{1}+n_{1} \varphi_{1}^{*}\right)\right\} \\
+e_{2}\left\{k_{1}\left[\alpha_{1}\left(u_{4}-u_{2}\right)+u_{4} u_{5}\right]+l_{1}\left(a_{1} v_{2}+b_{1} v_{4} v_{5}\right)\right. \\
-m_{1}\left[\sigma_{3}\left(w_{4}-w_{2}+\delta_{3} w_{1}\right)\right] \\
-n_{1}\left(\mu_{4}-\alpha_{4} \mu_{2}+\beta_{4} \mu_{4} \mu_{5}\right) \\
\left.-\left(m_{1} \varphi_{2}+n_{1} \varphi_{2}^{*}\right)\right\} \\
+e_{3}\left\{k_{2}\left(\gamma_{1} u_{1}-u_{5} u_{1}-u_{3}\right)+l_{2}\left(a_{2} v_{3}+b_{2} v_{1} v_{5}\right)\right. \\
-m_{2}\left[\left(\alpha_{3}-w_{5}\right) w_{1}-w_{3}-\delta_{3} w_{4}\right]
\end{gathered}
$$

$$
\begin{aligned}
& -n_{2}\left(\gamma_{4} \mu_{3}-\mu_{1} \mu_{5}+\mu_{5}\right) \\
& \left.-\left(m_{2} \varphi_{3}+n_{2} \varphi_{3}^{*}\right)\right\}
\end{aligned}
$$$$
+e_{4}\left\{k_{2}\left(\gamma_{1} u_{2}-u_{5} u_{2}-u_{4}\right)+l_{2}\left(a_{2} v_{4}+b_{2} v_{2} v_{5}\right)\right.
$$$$
-m_{2}\left[\left(\alpha_{3}-w_{5}\right) w_{2}-w_{4}-\delta_{3} w_{3}\right]
$$$$
\left.-n_{2}\left(\gamma_{4} \mu_{4}-\mu_{2} \mu_{5}\right)-\left(m_{2} \varphi_{4}+n_{2} \varphi_{4}^{*}\right)\right\}
$$$$
+e_{5}\left\{k_{3}\left(-\beta_{1} u_{5}+u_{1} u_{3}+u_{2} u_{4}\right)\right.
$$$$
+l_{3}\left[a_{3} v_{5}+b_{3}\left(v_{1} v_{3}+v_{2} v_{4}\right)\right]
$$$$
-m_{3}\left(-\beta_{3} w_{5}+w_{1} w_{3}+w_{2} w_{4}\right)
$$$$
-n_{3}\left[\delta_{4}\left(\mu_{1} \mu_{3}+\mu_{2} \mu_{4}\right)-\sigma_{4} \mu_{5}\right]
$$$$
\left.-\left(m_{3} \varphi_{5}+n_{3} \varphi_{5}^{*}\right)\right\} \text {. }
$$ 
Substituting (14) into (16) leads to

$$
\begin{aligned}
\dot{V}=e_{1}\left\{k_{1}\left[\alpha_{1}\left(u_{3}-u_{1}\right)+u_{3} u_{5}\right]\right. \\
+l_{1}\left(a_{1} v_{1}+b_{1} v_{3} v_{5}\right) \\
-m_{1}\left[\sigma_{3}\left(w_{3}-w_{1}-\delta_{3} w_{2}\right)\right] \\
-n_{1}\left(\mu_{3}-\alpha_{4} \mu_{1}+\beta_{4} \mu_{3} \mu_{5}\right) \\
-\left[k_{1} u_{1}+l_{1} v_{1}-m_{1} w_{1}-n_{1} \mu_{1}\right. \\
+a_{1}\left(k_{1} u_{2}+l_{1} v_{2}-m_{1} w_{2}-n_{1} \mu_{2}\right) \\
+k_{1}\left[\alpha_{1}\left(u_{3}-u_{1}\right)+u_{3} u_{5}\right] \\
+l_{1}\left(a_{1} v_{1}+b_{1} v_{3} v_{5}\right) \\
-m_{1}\left[\sigma_{3}\left(w_{3}-w_{1}-\delta_{3} w_{2}\right)\right] \\
\left.\left.\quad-n_{1}\left(\mu_{3}-\alpha_{4} \mu_{1}+\beta_{4} \mu_{3} \mu_{5}\right)\right]\right\}
\end{aligned}
$$$$
+e_{2}\left\{k_{1}\left[\alpha_{1}\left(u_{4}-u_{2}\right)+u_{4} u_{5}\right]\right.
$$$$
+l_{1}\left(a_{1} v_{2}+b_{1} v_{4} v_{5}\right)
$$$$
-m_{1}\left[\sigma_{3}\left(w_{4}-w_{2}+\delta_{3} w_{1}\right)\right]
$$$$
-n_{1}\left(\mu_{4}-\alpha_{4} \mu_{2}+\beta_{4} \mu_{4} \mu_{5}\right)
$$$$
-\left[k_{1} u_{2}+l_{1} v_{2}-m_{1} w_{2}-n_{1} \mu_{2}\right.
$$$$
-a_{1}\left(k_{1} u_{1}+l_{1} v_{1}-m_{1} w_{1}-n_{1} \mu_{1}\right)
$$$$
-a_{2}\left(k_{2} u_{3}+l_{2} v_{3}-m_{2} w_{3}-n_{2} \mu_{3}\right)
$$$$
+k_{1}\left[\alpha_{1}\left(u_{4}-u_{2}\right)+u_{4} u_{5}\right]
$$$$
+l_{1}\left(a_{1} v_{2}+b_{1} v_{4} v_{5}\right)
$$$$
-m_{1}\left[\sigma_{3}\left(w_{4}-w_{2}+\delta_{3} w_{1}\right)\right]
$$$$
\left.\left.-n_{1}\left(\mu_{4}-\alpha_{4} \mu_{2}+\beta_{4} \mu_{4} \mu_{5}\right)\right]\right\}
$$$$
+e_{3}\left\{k_{2}\left(\gamma_{1} u_{1}-u_{5} u_{1}-u_{3}\right)+l_{2}\left(a_{2} v_{3}+b_{2} v_{1} v_{5}\right)\right.
$$$$
-m_{2}\left[\left(\alpha_{3}-w_{5}\right) w_{1}-w_{3}-\delta_{3} w_{4}\right]
$$$$
-n_{2}\left(\gamma_{4} \mu_{3}-\mu_{1} \mu_{5}+\mu_{5}\right)
$$$$
-\left[k_{2} u_{3}+l_{2} v_{3}-m_{2} w_{3}-n_{2} \mu_{3}\right.
$$$$
+a_{2}\left(k_{1} u_{2}+l_{1} v_{2}-m_{1} w_{2}-n_{1} \mu_{2}\right)
$$$$
+a_{3}\left(k_{2} u_{4}+l_{2} v_{4}-m_{2} w_{4}-n_{2} \mu_{4}\right)
$$$$
+k_{2}\left(\gamma_{1} u_{1}-u_{5} u_{1}-u_{3}\right)
$$$$
+l_{2}\left(a_{2} v_{3}+b_{2} v_{1} v_{5}\right)
$$$$
-m_{2}\left[\left(\alpha_{3}-w_{5}\right) w_{1}-w_{3}-\delta_{3} w_{4}\right]
$$$$
\left.\left.-n_{2}\left(\gamma_{4} \mu_{3}-\mu_{1} \mu_{5}+\mu_{5}\right)\right]\right\}
$$

$$
\begin{aligned}
+e_{4}\left\{k_{2}\left(\gamma_{1} u_{2}-u_{5} u_{2}-u_{4}\right)+l_{2}\left(a_{2} v_{4}+b_{2} v_{2} v_{5}\right)\right. \\
-m_{2}\left[\left(\alpha_{3}-w_{5}\right) w_{2}-w_{4}-\delta_{3} w_{3}\right] \\
-n_{2}\left(\gamma_{4} \mu_{4}-\mu_{2} \mu_{5}\right) \\
-\left[k_{2} u_{4}+l_{2} v_{4}-m_{2} w_{4}-n_{2} \mu_{4}\right. \\
-a_{3}\left(k_{2} u_{3}+l_{2} v_{3}-m_{2} w_{3}-n_{2} \mu_{3}\right) \\
-b_{1}\left(k_{3} u_{5}+l_{3} v_{5}-m_{3} w_{5}-n_{3} \mu_{5}\right) \\
+k_{2}\left(\gamma_{1} u_{2}-u_{5} u_{2}-u_{4}\right) \\
+l_{2}\left(a_{2} v_{4}+b_{2} v_{2} v_{5}\right) \\
-m_{2}\left[\left(\alpha_{3}-w_{5}\right) w_{2}-w_{4}-\delta_{3} w_{3}\right] \\
\left.\left.\quad-n_{2}\left(\gamma_{4} \mu_{4}-\mu_{2} \mu_{5}\right)\right]\right\}
\end{aligned}
$$$$
+e_{5}\left\{k_{3}\left(-\beta_{1} u_{5}+u_{1} u_{3}+u_{2} u_{4}\right)\right.
$$$$
+l_{3}\left[a_{3} v_{5}+b_{3}\left(v_{1} v_{3}+v_{2} v_{4}\right)\right]
$$$$
-m_{3}\left(-\beta_{3} w_{5}+w_{1} w_{3}+w_{2} w_{4}\right)
$$$$
-n_{3}\left[\delta_{4}\left(\mu_{1} \mu_{3}+\mu_{2} \mu_{4}\right)-\sigma_{4} \mu_{5}\right]
$$$$
\text { - }\left[k_{3} u_{5}+l_{3} v_{5}-m_{3} w_{5}-n_{3} \mu_{5}\right.
$$$$
+b_{1}\left(k_{2} u_{4}+l_{2} v_{4}-m_{2} w_{4}-n_{2} \mu_{4}\right)
$$$$
+k_{3}\left(-\beta_{1} u_{5}+u_{1} u_{3}+u_{2} u_{4}\right)
$$$$
+l_{3}\left[a_{3} v_{5}+b_{3}\left(v_{1} v_{3}+v_{2} v_{4}\right)\right]
$$$$
-m_{3}\left(-\beta_{3} w_{5}+w_{1} w_{3}+w_{2} w_{4}\right)
$$$$
\left.\left.-n_{3}\left(\delta_{4}\left(\mu_{1} \mu_{3}+\mu_{2} \mu_{4}\right)-\sigma_{4} \mu_{5}\right)\right]\right\} \text {, }
$$

and we get

$$
\begin{aligned}
\dot{V}= & e_{1}\left(-e_{1}-a_{1} e_{2}\right)+e_{2}\left(-e_{2}+a_{1} e_{1}+a_{2} e_{3}\right) \\
& +e_{3}\left(-e_{3}-a_{2} e_{2}-a_{3} e_{4}\right) \\
& +e_{4}\left(-e_{4}+a_{3} e_{3}+b_{1} e_{5}\right)+e_{5}\left(-e_{5}-b_{1} e_{4}\right) \\
= & -e_{1}^{2}-e_{2}^{2}-e_{3}^{2}-e_{4}^{2}-e_{5}^{2} .
\end{aligned}
$$

Since $\dot{V} \leq 0$ as $t \rightarrow \infty$, according to the Lyapunov stability theory, we know $e_{i} \rightarrow 0(i=1,2,3,4,5)$; that is, $\lim _{t \rightarrow \infty}\|e\|=0$. Therefore, the drive systems (6) and (7) will achieve combination-combination synchronization with the response systems (8) and (9).

This completes the proof.

If we choose specific values of $k_{i}, l_{i}, m_{i}$, and $n_{i}(i=1,2,3)$, then we can have the following corollaries. 
Corollary 4. (i) Suppose that $n_{1}=n_{2}=n_{3}=0$, and if the controllers are chosen as follows:

$$
\begin{aligned}
\varphi_{1}=\frac{1}{m_{1}}\{ & k_{1} u_{1}+l_{1} v_{1}-m_{1} w_{1} \\
& +a_{1}\left(k_{1} u_{2}+l_{1} v_{2}-m_{1} w_{2}\right) \\
& +k_{1}\left[\alpha_{1}\left(u_{3}-u_{1}\right)+u_{3} u_{5}\right] \\
& +l_{1}\left(a_{1} v_{1}+b_{1} v_{3} v_{5}\right) \\
& \left.-m_{1} \sigma_{3}\left(w_{3}-w_{1}-\delta_{3} w_{2}\right)\right\},
\end{aligned}
$$

$\varphi_{2}=\frac{1}{m_{1}}\left\{k_{1} u_{2}+l_{1} v_{2}-m_{1} w_{2}\right.$

$$
-a_{1}\left(k_{1} u_{1}+l_{1} v_{1}-m_{1} w_{1}\right)
$$$$
-a_{2}\left(k_{2} u_{3}+l_{2} v_{3}-m_{2} w_{3}\right)
$$$$
+k_{1}\left[\alpha_{1}\left(u_{4}-u_{2}\right)+u_{4} u_{5}\right]
$$$$
+l_{1}\left(a_{1} v_{2}+b_{1} v_{4} v_{5}\right)
$$$$
\left.-m_{1} \sigma_{3}\left(w_{4}-w_{2}+\delta_{3} w_{1}\right)\right\}
$$

$$
\begin{aligned}
\varphi_{3}=\frac{1}{m_{2}}\{ & k_{2} u_{3}+l_{2} v_{3}-m_{2} w_{3} \\
& +a_{2}\left(k_{1} u_{2}+l_{1} v_{2}-m_{1} w_{2}\right) \\
& +a_{3}\left(k_{2} u_{4}+l_{2} v_{4}-m_{2} w_{4}\right) \\
& +k_{2}\left(\gamma_{1} u_{1}-u_{5} u_{1}-u_{3}\right) \\
& +l_{2}\left(a_{2} v_{3}+b_{2} v_{1} v_{5}\right) \\
& \left.-m_{2}\left[\left(\alpha_{3}-w_{5}\right) w_{1}-w_{3}-\delta_{3} w_{4}\right]\right\},
\end{aligned}
$$$$
\varphi_{4}=\frac{1}{m_{2}}\left\{k_{2} u_{4}+l_{2} v_{4}-m_{2} w_{4}\right.
$$$$
-a_{3}\left(k_{2} u_{3}+l_{2} v_{3}-m_{2} w_{3}\right)
$$$$
-b_{1}\left(k_{3} u_{5}+l_{3} v_{5}-m_{3} w_{5}\right)
$$$$
+k_{2}\left(\gamma_{1} u_{2}-u_{5} u_{2}-u_{4}\right)
$$$$
+l_{2}\left(a_{2} v_{4}+b_{2} v_{2} v_{5}\right)
$$$$
\left.-m_{2}\left[\left(\alpha_{3}-w_{5}\right) w_{2}-w_{4}-\delta_{3} w_{3}\right]\right\},
$$

$$
\begin{aligned}
\varphi_{5}=\frac{1}{m_{3}}\{ & k_{3} u_{5}+l_{3} v_{5}-m_{3} w_{5} \\
& +b_{1}\left(k_{2} u_{4}+l_{2} v_{4}-m_{2} w_{4}\right) \\
& +k_{3}\left(-\beta_{1} u_{5}+u_{1} u_{3}+u_{2} u_{4}\right) \\
& +l_{3}\left[a_{3} v_{5}+b_{3}\left(v_{1} v_{3}+v_{2} v_{4}\right)\right] \\
& \left.-m_{3}\left(-\beta_{3} w_{5}+w_{1} w_{3}+w_{2} w_{4}\right)\right\},
\end{aligned}
$$

then the drive systems (6) and (7) will achieve combination synchronization with the response system (8). (ii) Suppose that $m_{1}=m_{2}=m_{3}=0$, and if the controllers are chosen as follows:

$$
\begin{aligned}
\varphi_{1}^{*}=\frac{1}{n_{1}}\left\{k_{1} u_{1}+l_{1} v_{1}-n_{1} \mu_{1}\right. \\
+a_{1}\left(k_{1} u_{2}+l_{1} v_{2}-n_{1} \mu_{2}\right) \\
+k_{1}\left[\alpha_{1}\left(u_{3}-u_{1}\right)+u_{3} u_{5}\right] \\
+l_{1}\left(a_{1} v_{1}+b_{1} v_{3} v_{5}\right) \\
\left.-n_{1}\left(\mu_{3}-\alpha_{4} \mu_{1}+\beta_{4} \mu_{3} \mu_{5}\right)\right\},
\end{aligned}
$$

$\varphi_{2}^{*}=\frac{1}{n_{1}}\left\{k_{1} u_{2}+l_{1} v_{2}-n_{1} \mu_{2}\right.$

$-a_{1}\left(k_{1} u_{1}+l_{1} v_{1}-n_{1} \mu_{1}\right)$

$-a_{2}\left(k_{2} u_{3}+l_{2} v_{3}-n_{2} \mu_{3}\right)$

$+k_{1}\left[\alpha_{1}\left(u_{4}-u_{2}\right)+u_{4} u_{5}\right]$

$+l_{1}\left(a_{1} v_{2}+b_{1} v_{4} v_{5}\right)$

$\left.-\mathrm{n}_{1}\left(\boldsymbol{\mu}_{4}-\boldsymbol{\alpha}_{4} \boldsymbol{\mu}_{2}+\boldsymbol{\beta}_{4} \boldsymbol{\mu}_{4} \boldsymbol{\mu}_{5}\right)\right\}$,

$\varphi_{3}^{*}=\frac{1}{n_{2}}\left\{k_{2} u_{3}+l_{2} v_{3}-n_{2} \mu_{3}\right.$

$+a_{2}\left(k_{1} u_{2}+l_{1} v_{2}-n_{1} \mu_{2}\right)$

$+a_{3}\left(k_{2} u_{4}+l_{2} v_{4}-n_{2} \mu_{4}\right)$

$+\mathbf{k}_{2}\left(\gamma_{1} \mathbf{u}_{1}-\mathbf{u}_{5} \mathbf{u}_{1}-\mathbf{u}_{3}\right)$

$+l_{2}\left(a_{2} v_{3}+b_{2} v_{1} v_{5}\right)$

$\left.-\mathbf{n}_{2}\left(\gamma_{4} \mu_{3}-\mu_{1} \mu_{5}+\mu_{5}\right)\right\}$,

$\varphi_{4}^{*}=\frac{1}{n_{2}}\left\{k_{2} u_{4}+l_{2} \mathbf{v}_{4}-n_{2} \mu_{4}\right.$

$-a_{3}\left(k_{2} u_{3}+l_{2} v_{3}-n_{2} \mu_{3}\right)$

$-b_{1}\left(k_{3} u_{5}+l_{3} v_{5}-n_{3} \mu_{5}\right)$

$+\mathbf{k}_{2}\left(\gamma_{1} \mathbf{u}_{2}-\mathbf{u}_{5} \mathbf{u}_{2}-\mathbf{u}_{4}\right)$

$+l_{2}\left(a_{2} v_{4}+b_{2} v_{2} v_{5}\right)$

$\left.-\mathbf{n}_{2}\left(\gamma_{4} \boldsymbol{\mu}_{4}-\boldsymbol{\mu}_{2} \boldsymbol{\mu}_{5}\right)\right\}$,

$$
\begin{aligned}
& \varphi_{5}^{*}=\frac{1}{n_{3}}\left\{k_{3} u_{5}+l_{3} v_{5}-n_{3} \mu_{5}\right. \\
&+b_{1}\left(k_{2} u_{4}+l_{2} v_{4}-n_{2} \mu_{4}\right) \\
&+k_{3}\left(-\beta_{1} u_{5}+u_{1} u_{3}+u_{2} u_{4}\right) \\
&+l_{3}\left[a_{3} v_{5}+b_{3}\left(v_{1} v_{3}+v_{2} v_{4}\right)\right] \\
&\left.-n_{3}\left[\delta_{4}\left(\mu_{1} \mu_{3}+\mu_{2} \mu_{4}\right)-\sigma_{4} \mu_{5}\right]\right\},
\end{aligned}
$$

then the drive systems (6) and (7) will achieve combination synchronization with the response system (9). 
Corollary 5. (i) Suppose that $k_{1}=k_{2}=k_{3}=0, n_{1}=n_{2}=$ $n_{3}=0$, and $m_{1}=m_{2}=m_{3}=1$, and if the controllers are chosen as follows:

$$
\begin{aligned}
\varphi_{1}= & l_{1} v_{1}-w_{1}+a_{1}\left(l_{1} v_{2}-w_{2}\right) \\
& +l_{1}\left(a_{1} v_{1}+b_{1} v_{3} v_{5}\right) \\
& -\sigma_{3}\left(w_{3}-w_{1}-\delta_{3} w_{2}\right), \\
\varphi_{2}= & l_{1} v_{2}-w_{2}-a_{1}\left(l_{1} v_{1}-w_{1}\right) \\
& -a_{2}\left(l_{2} v_{3}-w_{3}\right)+l_{1}\left(a_{1} v_{2}+b_{1} v_{4} v_{5}\right) \\
& -\sigma_{3}\left(w_{4}-w_{2}+\delta_{3} w_{1}\right), \\
\varphi_{3}= & l_{2} v_{3}-w_{3}+a_{2}\left(l_{1} v_{2}-w_{2}\right) \\
& +a_{3}\left(l_{2} v_{4}-w_{4}\right)+l_{2}\left(a_{2} v_{3}+b_{2} v_{1} v_{5}\right) \\
& -\left[\left(\alpha_{3}-w_{5}\right) w_{1}-w_{3}-\delta_{3} w_{4}\right], \\
\varphi_{4}= & l_{2} v_{4}-w_{4}-a_{3}\left(l_{2} v_{3}-w_{3}\right) \\
& -b_{1}\left(l_{3} v_{5}-w_{5}\right)+l_{2}\left(a_{2} v_{4}+b_{2} v_{2} v_{5}\right) \\
& -\left[\left(\alpha_{3}-w_{5}\right) w_{2}-w_{4}-\delta_{3} w_{3}\right], \\
\varphi_{5}= & l_{3} v_{5}-w_{5}+b_{1}\left(l_{2} v_{4}-w_{4}\right) \\
& +l_{3}\left[a_{3} v_{5}+b_{3}\left(v_{1} v_{3}+v_{2} v_{4}\right)\right] \\
& -\left(-\beta_{3} w_{5}+w_{1} w_{3}+w_{2} w_{4}\right),
\end{aligned}
$$

then the drive system (7) will achieve projective synchronization with the response system (8).

(ii) Suppose that $l_{1}=l_{2}=l_{3}=0, n_{1}=n_{2}=n_{3}=0$, and $m_{1}=m_{2}=m_{3}=1$, and if the controllers are chosen as follows:

$$
\begin{aligned}
\varphi_{1}= & k_{1} u_{1}-w_{1}+\alpha_{3}\left(k_{1} u_{2}-w_{2}\right) \\
& +k_{1}\left[\alpha_{1}\left(u_{3}-u_{1}\right)+u_{3} u_{5}\right] \\
& -\sigma_{3}\left(w_{3}-w_{1}-\delta_{3} w_{2}\right) \\
\varphi_{2}= & k_{1} u_{2}-w_{2}-\alpha_{3}\left(k_{1} u_{1}-w_{1}\right) \\
& -\beta_{3}\left(k_{2} u_{3}-w_{3}\right)+k_{1}\left[\alpha_{1}\left(u_{4}-u_{2}\right)+u_{4} u_{5}\right] \\
& -\sigma_{3}\left(w_{4}-w_{2}+\delta_{3} w_{1}\right) \\
\varphi_{3}= & k_{2} u_{3}-w_{3}+\beta_{3}\left(k_{1} u_{2}-w_{2}\right) \\
& +\delta_{3}\left(k_{2} u_{4}-w_{4}\right)+k_{2}\left(\gamma_{1} u_{1}-u_{5} u_{1}-u_{3}\right) \\
& -\left[\left(\alpha_{3}-w_{5}\right) w_{1}-w_{3}-\delta_{3} w_{4}\right], \\
\varphi_{4}= & k_{2} u_{4}-w_{4}-\delta_{3}\left(k_{2} u_{3}-w_{3}\right) \\
& -\sigma_{3}\left(k_{3} u_{5}-w_{5}\right)+k_{2}\left(\gamma_{1} u_{2}-u_{5} u_{2}-u_{4}\right) \\
& -\left[\left(\alpha_{3}-w_{5}\right) w_{2}-w_{4}-\delta_{3} w_{3}\right], \\
\varphi_{5}= & k_{3} u_{5}-w_{5}+\sigma_{3}\left(k_{2} u_{4}-w_{4}\right) \\
& +k_{3}\left(-\beta_{1} u_{5}+u_{1} u_{3}+u_{2} u_{4}\right) \\
& -\left(-\beta_{3} w_{5}+w_{1} w_{3}+w_{2} w_{4}\right),
\end{aligned}
$$

then the drive system (6) will achieve projective synchronization with the response system (8). (iii) Suppose that $k_{1}=k_{2}=k_{3}=0, m_{1}=m_{2}=m_{3}=0$, and $n_{1}=n_{2}=n_{3}=1$, and if the controllers are chosen as follows:

$$
\begin{aligned}
\varphi_{1}^{*}= & l_{1} v_{1}-\mu_{1}+a_{1}\left(l_{1} v_{2}-\mu_{2}\right) \\
& +l_{1}\left(a_{1} v_{1}+b_{1} v_{3} v_{5}\right) \\
& -\left(\mu_{3}-\alpha_{4} \mu_{1}+\beta_{4} \mu_{3} \mu_{5}\right), \\
\varphi_{2}^{*}= & l_{1} v_{2}-\mu_{2}-a_{1}\left(l_{1} v_{1}-\mu_{1}\right) \\
& -a_{2}\left(l_{2} v_{3}-\mu_{3}\right)+l_{1}\left(a_{1} v_{2}+b_{1} v_{4} v_{5}\right) \\
& -\left(\mu_{4}-\alpha_{4} \mu_{2}+\beta_{4} \mu_{4} \mu_{5}\right), \\
\varphi_{3}^{*}= & l_{2} v_{3}-\mu_{3}+a_{2}\left(l_{1} v_{2}-\mu_{2}\right) \\
& +a_{3}\left(l_{2} v_{4}-\mu_{4}\right)+l_{2}\left(a_{2} v_{3}+b_{2} v_{1} v_{5}\right) \\
& -\left(\gamma_{4} \mu_{3}-\mu_{1} \mu_{5}+\mu_{5}\right), \\
\varphi_{4}^{*}= & l_{2} v_{4}-\mu_{4}-a_{3}\left(l_{2} v_{3}-\mu_{3}\right) \\
& -b_{1}\left(l_{3} v_{5}-\mu_{5}\right)+l_{2}\left(a_{2} v_{4}+b_{2} v_{2} v_{5}\right) \\
& -\left(\gamma_{4} \mu_{4}-\mu_{2} \mu_{5}\right), \\
\varphi_{5}^{*}= & l_{3} v_{5}-\mu_{5}+b_{1}\left(l_{2} v_{4}-\mu_{4}\right) \\
& +l_{3}\left[a_{3} v_{5}+b_{3}\left(v_{1} v_{3}+v_{2} v_{4}\right)\right] \\
& -\left(\delta_{4}\left(\mu_{1} \mu_{3}+\mu_{2} \mu_{4}\right)-\sigma_{4} \mu_{5}\right),
\end{aligned}
$$

then the drive system (7) will achieve projective synchronization with the response system (9).

(iv) Suppose that $l_{1}=l_{2}=l_{3}=0, m_{1}=m_{2}=m_{3}=0$, and $n_{1}=n_{2}=n_{3}=1$, and if the controllers are chosen as follows:

$$
\begin{aligned}
\varphi_{1}^{*}= & k_{1} u_{1}-\mu_{1}+\alpha_{3}\left(k_{1} u_{2}-\mu_{2}\right) \\
& +k_{1}\left[\alpha_{1}\left(u_{3}-u_{1}\right)+u_{3} u_{5}\right] \\
& -\left(\mu_{3}-\alpha_{4} \mu_{1}+\beta_{4} \mu_{3} \mu_{5}\right), \\
\varphi_{2}^{*}= & k_{1} u_{2}-\mu_{2}-\alpha_{3}\left(k_{1} u_{1}-\mu_{1}\right) \\
& -\beta_{3}\left(k_{2} u_{3}-\mu_{3}\right) \\
& +k_{1}\left[\alpha_{1}\left(u_{4}-u_{2}\right)+u_{4} u_{5}\right] \\
& -\left(\mu_{4}-\alpha_{4} \mu_{2}+\beta_{4} \mu_{4} \mu_{5}\right), \\
\varphi_{3}^{*}= & k_{2} u_{3}-\mu_{3}+\beta_{3}\left(k_{1} u_{2}-\mu_{2}\right) \\
& +\delta_{3}\left(k_{2} u_{4}-\mu_{4}\right) \\
& +k_{2}\left(\gamma_{1} u_{1}-u_{5} u_{1}-u_{3}\right) \\
& -\left(\gamma_{4} \mu_{3}-\mu_{1} \mu_{5}+\mu_{5}\right),
\end{aligned}
$$




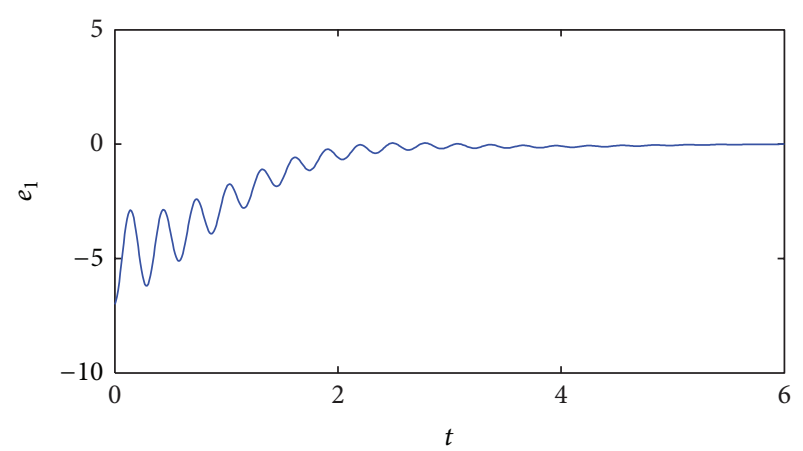

(a)

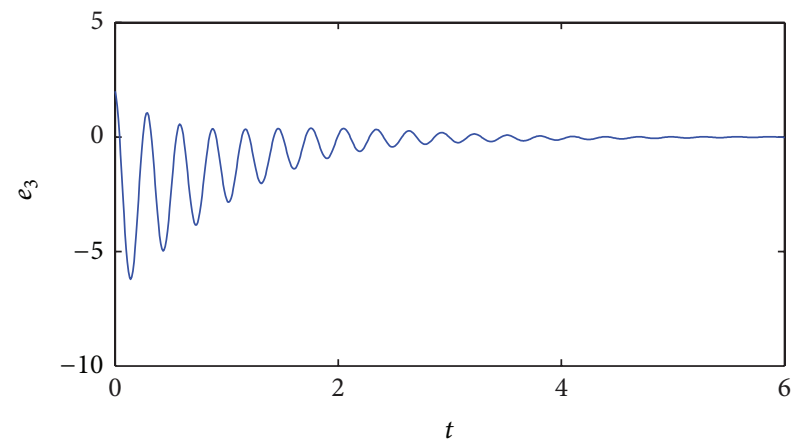

(c)

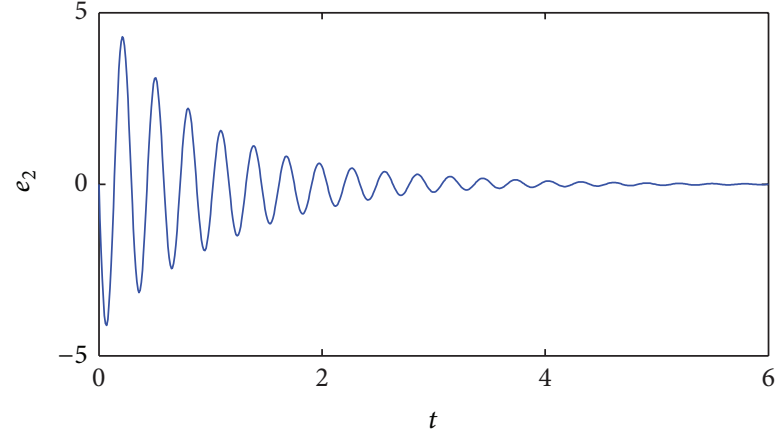

(b)

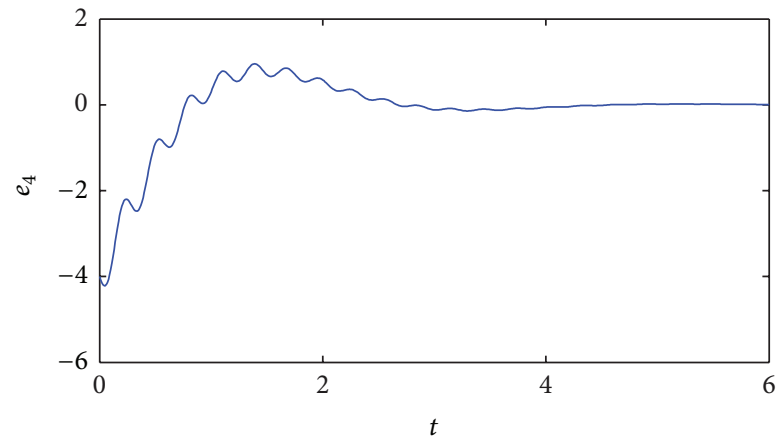

(d)

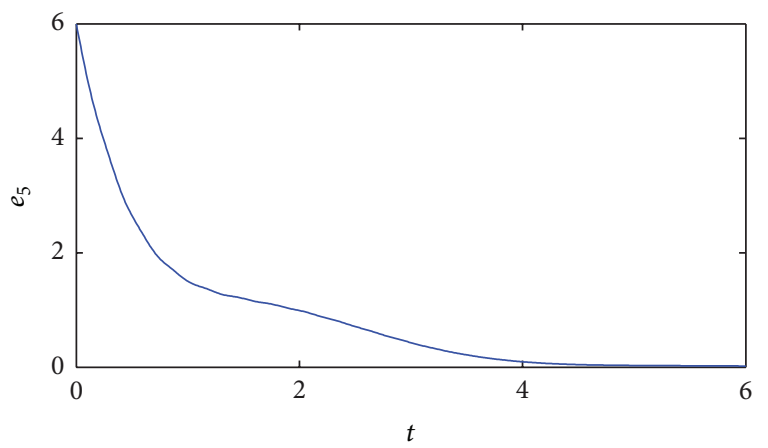

(e)

Figure 5: Combination-combination synchronization errors $e_{1}, e_{2}, e_{3}, e_{4}$, and $e_{5}$ between the drive systems (6) and (7) and the response systems (8) and (9), where $e_{i}=u_{i}+v_{i}-w_{i}-\mu_{i}(i=1,2,3,4,5)$.

$$
\begin{aligned}
\varphi_{4}^{*}= & k_{2} u_{4}-\mu_{4}-\delta_{3}\left(k_{2} u_{3}-\mu_{3}\right) \\
& -\sigma_{3}\left(k_{3} u_{5}-\mu_{5}\right)+k_{2}\left(\gamma_{1} u_{2}-u_{5} u_{2}-u_{4}\right) \\
& -\left(\gamma_{4} \mu_{4}-\mu_{2} \mu_{5}\right), \\
\varphi_{5}^{*}= & k_{3} u_{5}-\mu_{5}+\sigma_{3}\left(k_{2} u_{4}-\mu_{4}\right) \\
& +k_{3}\left(-\beta_{1} u_{5}+u_{1} u_{3}+u_{2} u_{4}\right) \\
& -\left(\delta_{4}\left(\mu_{1} \mu_{3}+\mu_{2} \mu_{4}\right)-\sigma_{4} \mu_{5}\right),
\end{aligned}
$$

then the drive system (6) will achieve projective synchronization with the response system (9).
Corollary 6. (i) Suppose that $k_{1}=k_{2}=k_{3}=0, l_{1}=l_{2}=l_{3}=$ $0, n_{1}=n_{2}=n_{3}=0$, and $m_{1}=m_{2}=m_{3}=1$, and if the controllers are chosen as follows:

$$
\begin{gathered}
\varphi_{1}=-w_{1}-\alpha_{3} w_{2}-\sigma_{3}\left(w_{3}-w_{1}-\delta_{3} w_{2}\right), \\
\varphi_{2}=-w_{2}+\alpha_{3} w_{1}+\beta_{3} w_{3}-\sigma_{3}\left(w_{4}-w_{2}+\delta_{3} w_{1}\right), \\
\varphi_{3}=-w_{3}-\beta_{3} w_{2}-\delta_{3} w_{4} \\
-\left[\left(\alpha_{3}-w_{5}\right) w_{1}-w_{3}-\delta_{3} w_{4}\right], \\
\varphi_{4}=-w_{4}+\delta_{3} w_{3}+\sigma_{3} w_{5} \\
-\left[\left(\alpha_{3}-w_{5}\right) w_{2}-w_{4}-\delta_{3} w_{3}\right], \\
\varphi_{5}=-w_{5}-\sigma_{3} w_{4}-\left(-\beta_{3} w_{5}+w_{1} w_{3}+w_{2} w_{4}\right),
\end{gathered}
$$

then system (8) is stabilized to the equilibrium $O(0,0,0,0,0)$. 


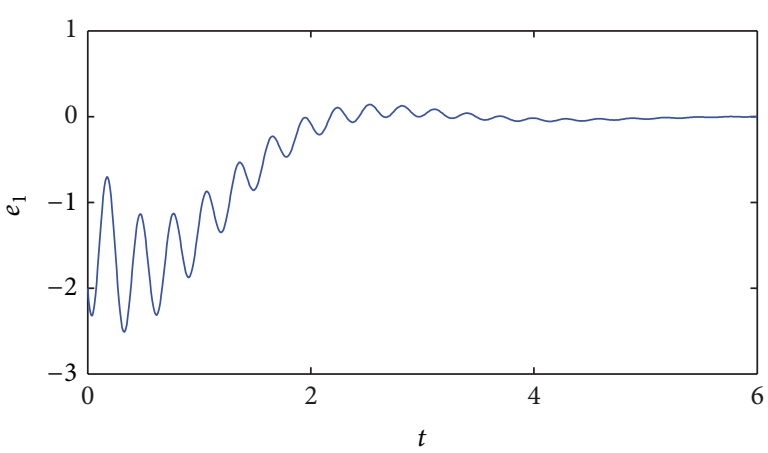

(a)

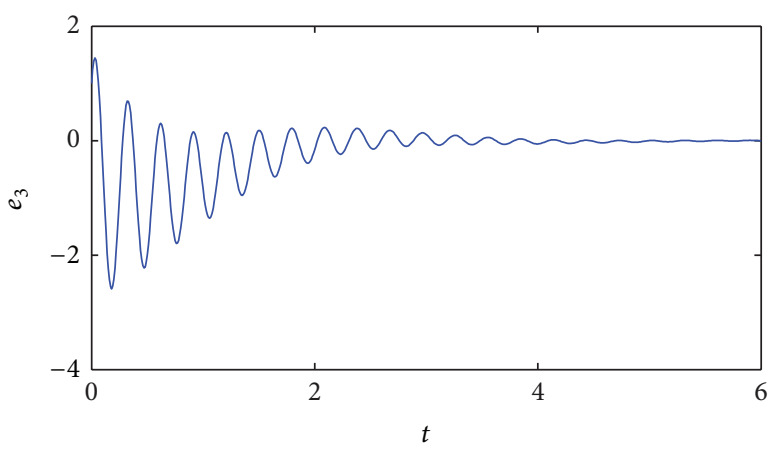

(c)

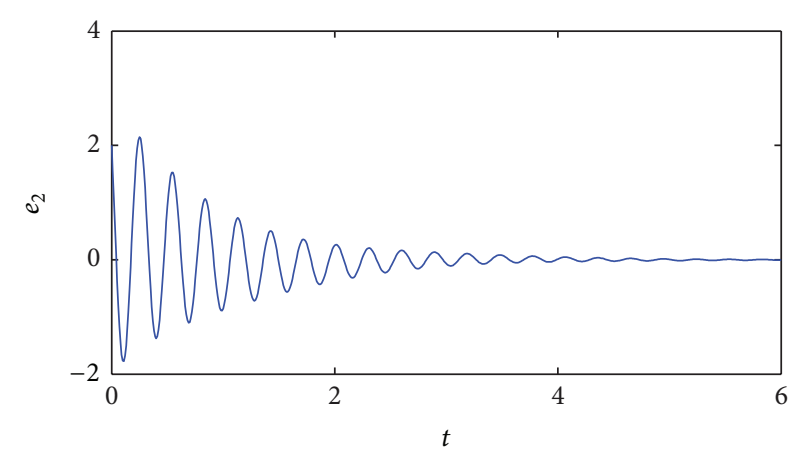

(b)

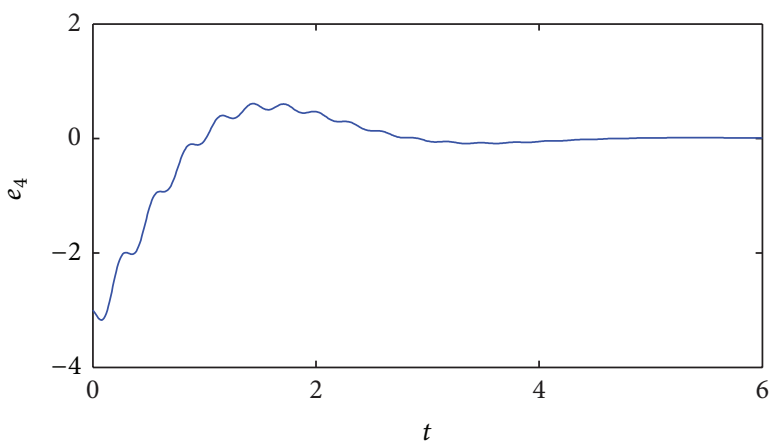

(d)

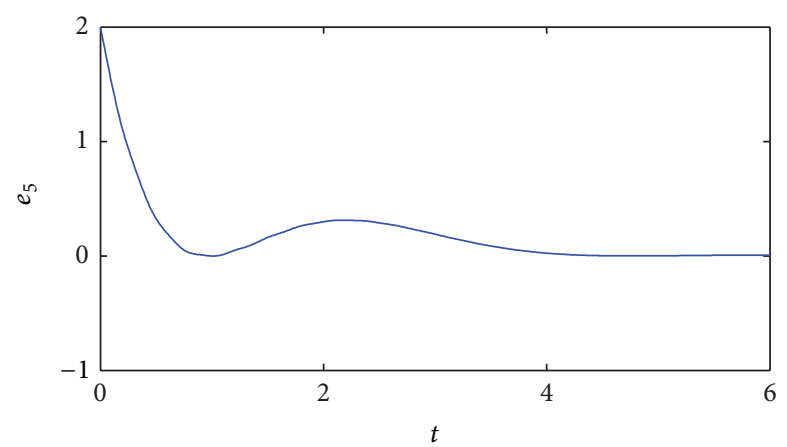

(e)

FIGURE 6: Combination synchronization errors $e_{1}, e_{2}, e_{3}, e_{4}$, and $e_{5}$ between the drive systems (6) and (7) and the response system (8), where $e_{i}=u_{i}+v_{i}-w_{i}(i=1,2,3,4,5)$.

(ii) Suppose that $k_{1}=k_{2}=k_{3}=0, l_{1}=l_{2}=l_{3}=0$, $m_{1}=m_{2}=m_{3}=0$, and $n_{1}=n_{2}=n_{3}=1$, and if the controllers are chosen as follows:

$$
\begin{gathered}
\varphi_{1}^{*}=-\mu_{1}-\alpha_{4} \mu_{2}-\left(\mu_{3}-\alpha_{4} \mu_{1}+\beta_{4} \mu_{3} \mu_{5}\right), \\
\varphi_{2}^{*}=-\mu_{2}+\alpha_{4} \mu_{1}+\beta_{4} \mu_{3}-\left(\mu_{4}-\alpha_{4} \mu_{2}+\beta_{4} \mu_{4} \mu_{5}\right), \\
\varphi_{3}^{*}=-\mu_{3}-\beta_{4} \mu_{2}-\gamma_{4} \mu_{4}-\left(\gamma_{4} \mu_{3}-\mu_{1} \mu_{5}+\mu_{5}\right), \\
\varphi_{4}^{*}=-\mu_{4}+\gamma_{4} \mu_{3}+\sigma_{4} \mu_{5}-\left(\gamma_{4} \mu_{4}-\mu_{2} \mu_{5}\right), \\
\varphi_{5}^{*}=-\mu_{5}-\sigma_{4} \mu_{4}-\left[\delta_{4}\left(\mu_{1} \mu_{3}+\mu_{2} \mu_{4}\right)-\sigma_{4} \mu_{5}\right],
\end{gathered}
$$

then system (9) is stabilized to the equilibrium $O(0,0,0,0,0)$.
Remark 7. The above corollaries can be easily obtained from Theorem 3, and their proofs are similar to that of Theorem 3, so we omit the proofs here.

\section{Numerical Simulations}

In this section, three numerical examples are presented to illustrate the theoretical analysis. In the following numerical simulations, the fourth-order Runge-kutta method is employed with time step size 0.001 . The system parameters are selected as $\alpha_{1}=30, \gamma_{1}=90, \beta_{1}=11, a_{1}=9.5, a_{2}=-19$, $a_{3}=-3, b_{1}=-1, b_{2}=1, b_{3}=1, \alpha_{3}=50.0625, \beta_{3}=0.75, \sigma_{3}=$ $5, \delta_{3}=0.25, \alpha_{4}=3.5, \beta_{4}=0.599, \gamma_{4}=3, \delta_{4}=2$, and $\sigma_{4}=9$, so that the four nonlinear complex chaotic systems exhibit chaotic behaviors, respectively. 


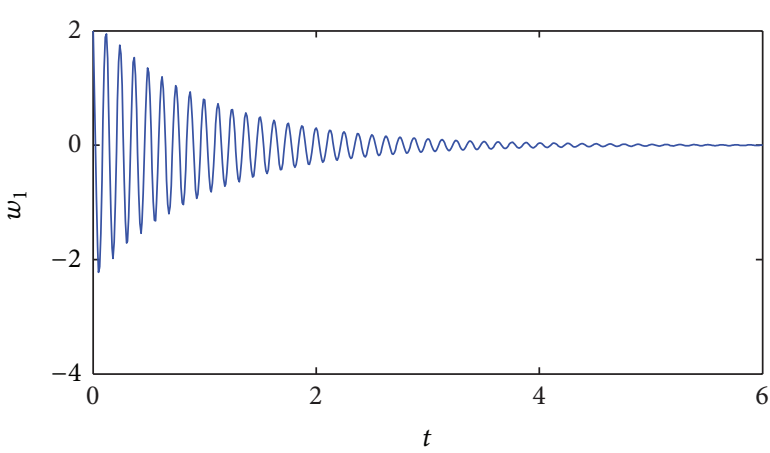

(a)

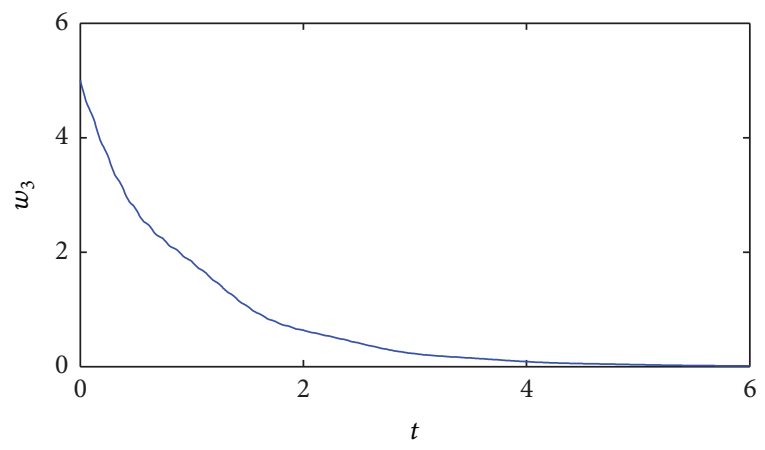

(c)

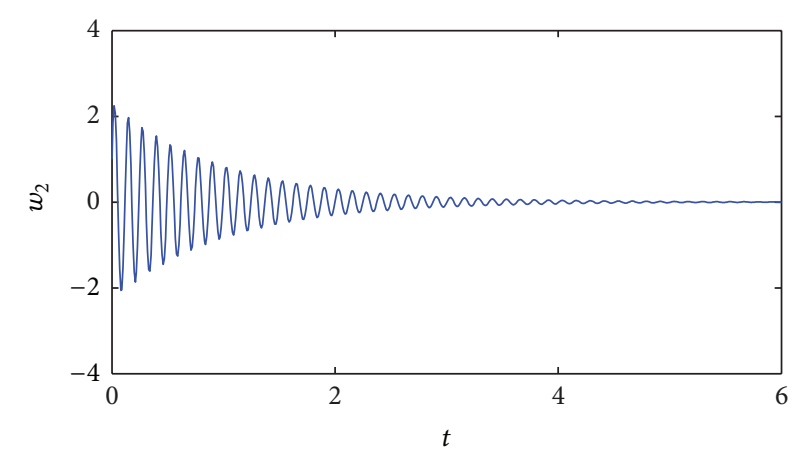

(b)

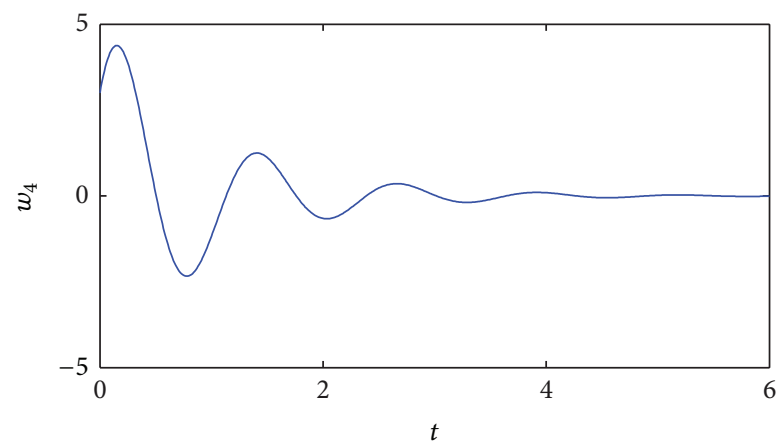

(d)

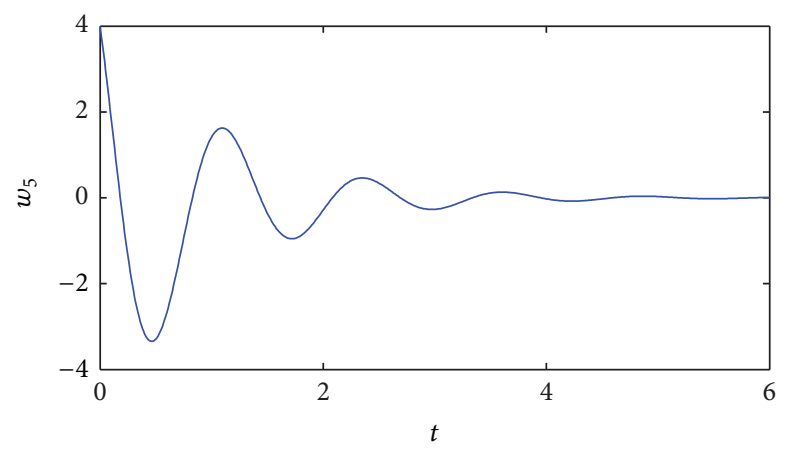

(e)

FIGURE 7: Time evolution of the states of system (8).

Firstly, consider the combination-combination synchronization of the two drive systems (6) and (7) and the response systems (8) and (9) with the controllers (14). We assume $k_{1}=k_{2}=k_{3}=1, l_{1}=l_{2}=l_{3}=1, m_{1}=m_{2}=m_{3}=1$, and $n_{1}=n_{2}=n_{3}=1$, and the initial states for the drive systems and response systems are arbitrarily given by $\left(x_{11}(0), x_{12}(0)\right.$, $\left.x_{13}(0)\right)=(2+4 i, 1+3 i, 2),\left(x_{21}(0), x_{22}(0), x_{23}(0)\right)=(-2-i$, $5-3 i, 4),\left(y_{11}(0), y_{12}(0), y_{13}(0)\right)=(2+i, 5+3 i, 4)$, and $\left(y_{21}(0)\right.$, $\left.y_{22}(0), y_{23}(0)\right)=(5+2 i,-1+i,-4)$; that is, $\left(u_{1}(0), u_{2}(0), u_{3}(0)\right.$, $\left.u_{4}(0), u_{5}(0)\right)=(2,4,1,3,2),\left(v_{1}(0), v_{2}(0), v_{3}(0), v_{4}(0), v_{5}(0)\right)=$ $(-2,-1,5,-3,4),\left(w_{1}(0), w_{2}(0), w_{3}(0), w_{4}(0), w_{5}(0)\right)=(2,1$, $5,3,4)$, and $\left(\mu_{1}(0), \mu_{2}(0), \mu_{3}(0), \mu_{4}(0), \mu_{5}(0)\right)=(5,2,-1,1$, $-4)$, respectively. The corresponding numerical results are shown in Figure 5. Figure 5 displays time response of the combination-combination synchronization errors $e_{1}, e_{2}, e_{3}$, $e_{4}$, and $e_{5}$, where $e_{i}=u_{i}+v_{i}-w_{i}-\mu_{i}(i=1,2,3,4,5)$. The errors converge to zero which implies that the drive systems (6) and (7) and the response systems (8) and (9) have achieved combination-combination synchronization.

Secondly, consider the combination synchronization of the two drive systems (6) and (7) and the response system (8) with the controllers (19). We assume $k_{1}=k_{2}=k_{3}=1, l_{1}=$ $l_{2}=l_{3}=1, m_{1}=m_{2}=m_{3}=1$, and $n_{1}=n_{2}=n_{3}=0$. The corresponding numerical results are shown in Figure 6. Figure 6 displays time response of the combination synchronization errors $e_{1}, e_{2}, e_{3}, e_{4}$, and $e_{5}$, where $e_{i}=u_{i}+v_{i}-w_{i}$ $(i=1,2,3,4,5)$. The errors converge to zero which implies that the drive systems (6) and (7) and the response system (8) have achieved combination synchronization.

Finally, consider another special case, that is, when $k_{1}=$ $k_{2}=k_{3}=0, l_{1}=l_{2}=l_{3}=0, m_{1}=m_{2}=m_{3}=1$, and $n_{1}=$ $n_{2}=n_{3}=0$, system (8) will be stabilized to its equilibrium 
$O(0,0,0,0,0)$. Figure 7 shows the time evolution of the states $w_{1}, w_{2}, w_{3}, w_{4}$, and $w_{5}$ of system (8) with controller (25), which illustrates that system (8) is stabilized to the equilibrium $O(0,0,0,0,0)$.

\section{Conclusions}

In this paper, we investigate the combination-combination synchronization of four nonlinear complex chaotic systems. Based on the Lyapunov stability theory, corresponding controllers to achieve combination-combination synchronization among four different nonlinear complex chaotic systems are derived. The special cases, such as combination synchronization and projective synchronization, are studied as well. This synchronization scheme has advantages over the usual drive-response synchronization, such as being able to provide greater security in secure communication. In [24], the authors applied combination synchronization in secure communication; the signal was divided into two parts, and each part was transmitted by a different chaotic system (the drive system), which implied that the signal transmitted by this model may have stronger antiattack ability and antitranslated capability than that transmitted by the usual transmission model. When applying the nonlinear complex systems in communications, the complex variables will double the number of variables and can increase the content and security of the transmitted information. Thus combination-combination synchronization of complex nonlinear systems can find better applications in security communication, such as wireless communication [25].

\section{Conflict of Interests}

The authors declare that there is no conflict of interests regarding the publication of this paper.

\section{Acknowledgments}

This work was supported by the Youth Foundation of Yunnan University of Nationalities under Grant no. 11QN07, the Natural Science Foundation of Yunnan Province under Grants no. 2009CD019 and no. 2011FZ172, and the Natural Science Foundation of China under Grant no. 61263042.

\section{References}

[1] A. C. Fowler, M. J. McGuinness, and J. D. Gibbon, “The complex Lorenz equations," Physica D, vol. 4, no. 2, pp. 139-163, 1981/82.

[2] C. Z. Ning and H. Haken, "Detuned lasers and the complex Lorenz equations: subcritical and supercritical Hopf bifurcations," Physical Review A, vol. 41, pp. 3826-3837, 1990.

[3] J. D. Gibbon and M. J. McGuinness, "The real and complex Lorenz equations in rotating fluids and lasers," Physica D, vol. 5, no. 1, pp. 108-122, 1982.

[4] G. M. Mahmoud and A. A. M. Farghaly, "Chaos control of chaotic limit cycles of real and complex van der Pol oscillators," Chaos, Solitons and Fractals, vol. 21, no. 4, pp. 915-924, 2004.

[5] G. M. Mahmoud, T. Bountis, and E. E. Mahmoud, "Active control and global synchronization of the complex Chen and
Lü systems," International Journal of Bifurcation and Chaos in Applied Sciences and Engineering, vol. 17, no. 12, pp. 4295-4308, 2007.

[6] G. M. Mahmoud, T. Bountis, M. A. Al-Kashif, and S. A. Aly, "Dynamical properties and synchronization of complex nonlinear equations for detuned lasers," Dynamical Systems, vol. 24, no. 1, pp. 63-79, 2009.

[7] E. E. Mahmoud, "Dynamics and synchronization of new hyperchaotic complex Lorenz system," Mathematical and Computer Modelling, vol. 55, no. 7-8, pp. 1951-1962, 2012.

[8] G. M. Mahmoud, M. E. Ahmed, and N. Sabor, "On autonomous and nonautonomous modified hyperchaotic complex Lü systems," International Journal of Bifurcation and Chaos in Applied Sciences and Engineering, vol. 21, no. 7, pp. 1913-1926, 2011.

[9] G. M. Mahmoud and M. E. Ahmed, "A hyperchaotic complex system generating two-, three-, and four-scroll attractors," Journal of Vibration and Control, vol. 18, no. 6, pp. 841-849, 2012.

[10] L. M. Pecora and T. L. Carroll, "Synchronization in chaotic systems," Physical Review Letters, vol. 64, no. 8, pp. 821-824, 1990.

[11] G. Chen and X. Dong, From Chaos to Order: Methodologies, Perspectives and Applications, vol. 24, World Scientific Publishing, Singapore, 1998.

[12] A. A. Koronovskii, O. I. Moskalenko, and A. E. Hramov, "On the use of chaotic synchronization for secure communication," Physics-Uspekhi, vol. 52, pp. 1213-1238, 2009.

[13] B. S. Dmitriev, A. E. Hramov, A. A. Koronovskii, A. V. Starodubov, D. I. Trubetskov, and Y. D. Zharkov, "First experimental observation of generalized synchronization phenomena in microwave oscillators," Physical Review Letters, vol. 102, Article ID 074101, 2009.

[14] M. Hu, Y. Yang, Z. Xu, and L. Guo, "Hybrid projective synchronization in a chaotic complex nonlinear system," Mathematics and Computers in Simulation, vol. 79, no. 3, pp. 449-457, 2008.

[15] S. Liu and P. Liu, "Adaptive anti-synchronization of chaotic complex nonlinear systems with unknown parameters," Nonlinear Analysis: Real World Applications, vol. 12, no. 6, pp. 30463055, 2011.

[16] E. E. Mahmoud, "Modified projective phase synchronization of chaotic complex nonlinear systems," Mathematics and Computers in Simulation, vol. 89, pp. 69-85, 2013.

[17] G. M. Mahmoud, E. E. Mahmoud, and A. A. Arafa, "On projective synchronization of hyperchaotic complex nonlinear systems based on passive theory for secure communications," Physica Scripta, vol. 87, no. 5, Article ID 055002, 2013.

[18] X. B. Zhou, M. R. Jiang, and X. M. Cai, "Synchronization of a novel hyperchaotic complex-variable system based on finitetime stability Theory," Entropy, vol. 15, pp. 4334-4344, 2013.

[19] R. Z. Luo, Y. L. Wang, and S. C. Deng, "Combination synchronization of three classic chaotic systems using active backstepping design,” Chaos, vol. 21, no. 4, Article ID 043114, 2011.

[20] X. B. Zhou, M. R. Jiang, and Y. Q. Huang, "Combination synchronization of three identical or different nonlinear complex hyperchaotic systems," Entropy, vol. 15, pp. 3746-3761, 2013.

[21] J. Sun, Y. Shen, G. Zhang, C. Xu, and G. Cui, "Combinationcombination synchronization among four identical or different chaotic systems," Nonlinear Dynamics, vol. 73, no. 3, pp. 12111222, 2013.

[22] G. M. Mahmoud, M. A. Al-Kashif, and A. A. Farghaly, "Chaotic and hyperchaotic attractors of a complex nonlinear system," Journal of Physics A, vol. 41, no. 5, Article ID 055104, 12 pages, 2008. 
[23] G. M. Mahmoud, S. A. Aly, and M. A. AL-Kashif, "Dynamical properties and chaos synchronization of a new chaotic complex nonlinear system," Nonlinear Dynamics, vol. 51, no. 1-2, pp. 171181, 2008.

[24] R. Z. Luo and Y. L. Wang, "Finite-time stochastic combination synchronization of three different chaotic systems and its application in secure communication," Chaos, vol. 22, no. 2, Article ID 023109, 2012.

[25] H. P. Ren, M. S. Baptista, and C. Grebogi, "Wireless communication with chaos," Physical Review Letters, vol. 110, no. 18, Article ID 184101, 5 pages, 2013. 


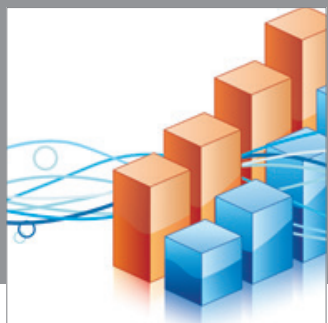

Advances in

Operations Research

mansans

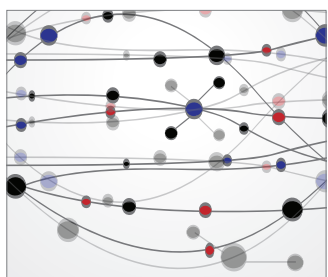

The Scientific World Journal
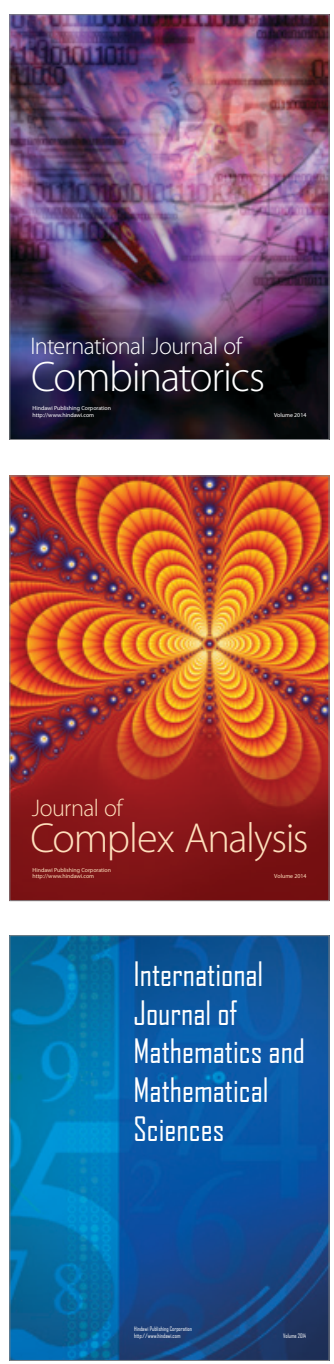
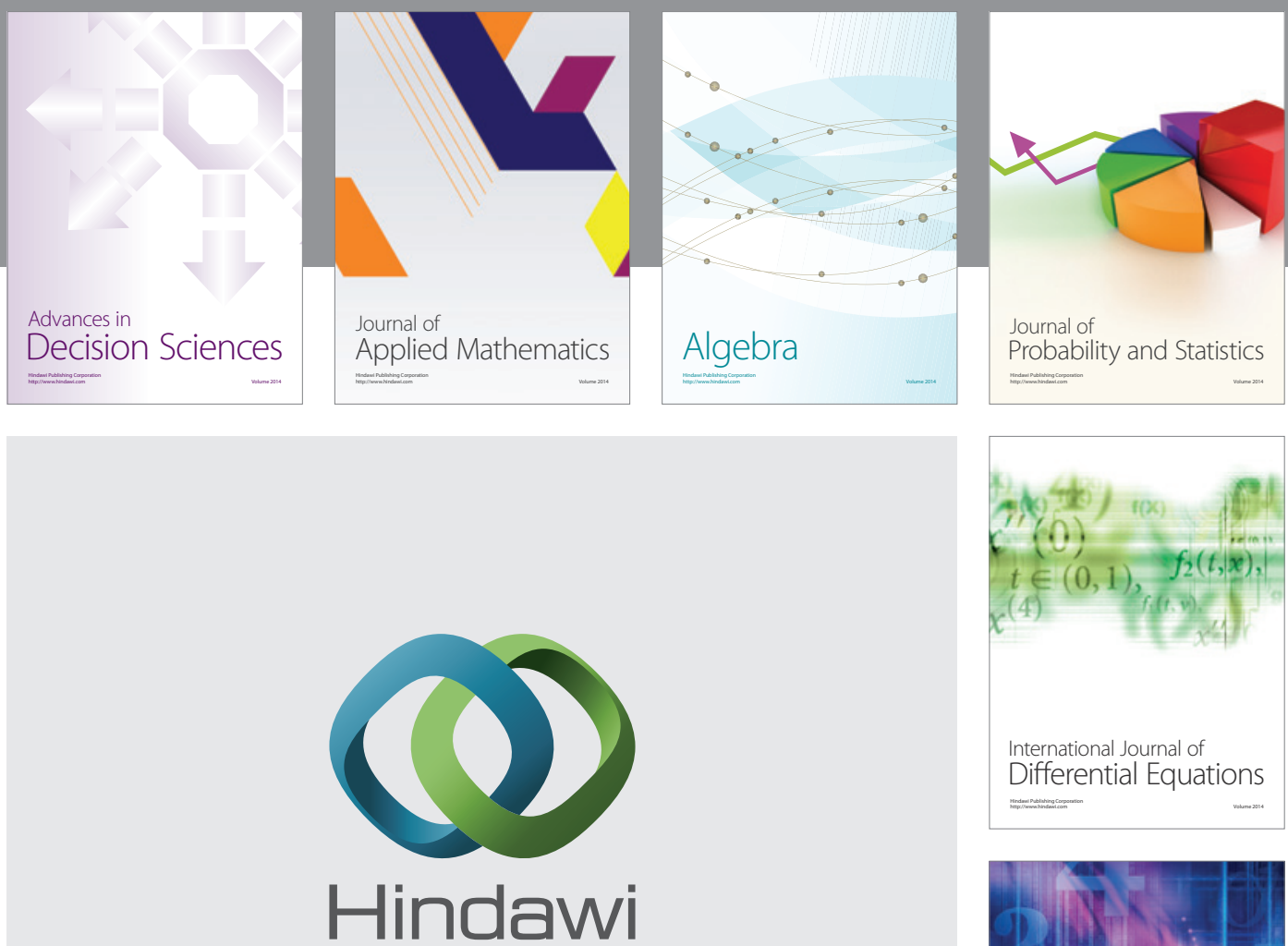

Submit your manuscripts at http://www.hindawi.com
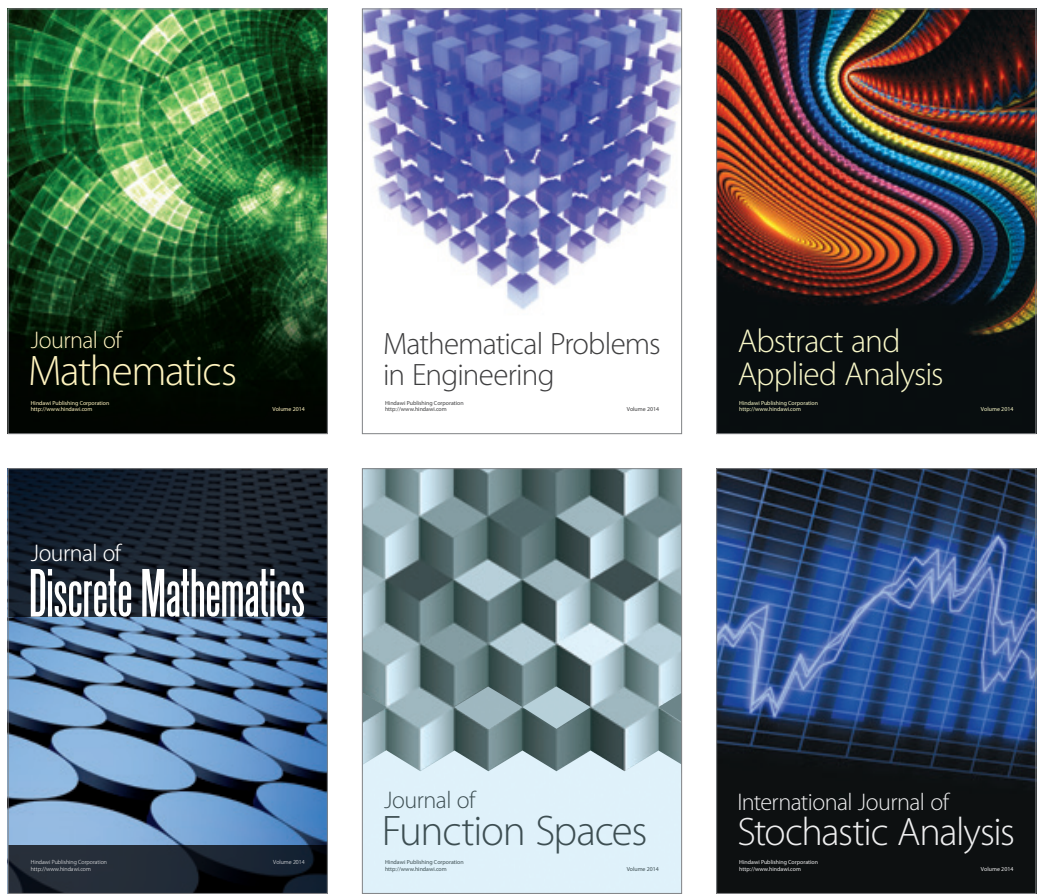

Journal of

Function Spaces

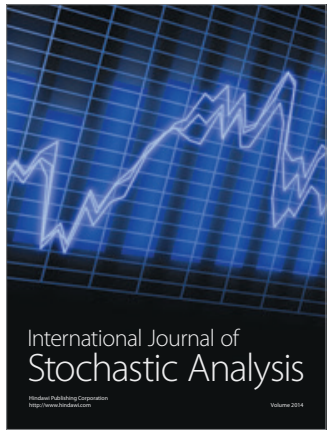

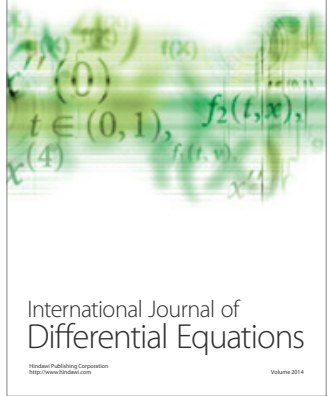
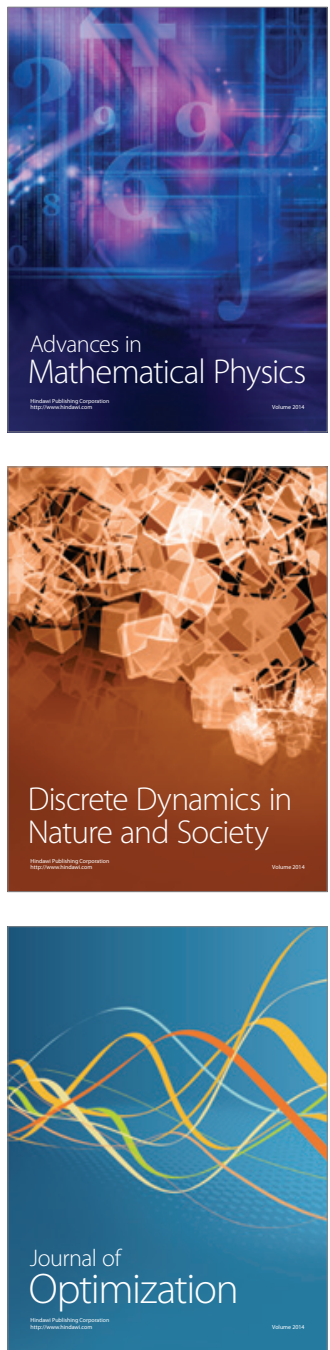